\title{
GLOBAL ATTRACTOR OF COUPLED DIFFERENCE EQUATIONS AND APPLICATIONS TO LOTKA-VOLTERRA SYSTEMS
}

C. V. PAO

Received 22 April 2004

This paper is concerned with a coupled system of nonlinear difference equations which is a discrete approximation of a class of nonlinear differential systems with time delays. The aim of the paper is to show the existence and uniqueness of a positive solution and to investigate the asymptotic behavior of the positive solution. Sufficient conditions are given to ensure that a unique positive equilibrium solution exists and is a global attractor of the difference system. Applications are given to three basic types of Lotka-Volterra systems with time delays where some easily verifiable conditions on the reaction rate constants are obtained for ensuring the global attraction of a positive equilibrium solution.

\section{Introduction}

Difference equations appear as discrete phenomena in nature as well as discrete analogues of differential equations which model various phenomena in ecology, biology, physics, chemistry, economics, and engineering. There are large amounts of works in the literature that are devoted to various qualitative properties of solutions of difference equations, such as existence-uniqueness of positive solutions, asymptotic behavior of solutions, stability and attractor of equilibrium solutions, and oscillation or nonoscillation of solutions (cf. $[1,4,11,13]$ and the references therein). In this paper, we investigate some of the above qualitative properties of solutions for a coupled system of nonlinear difference equations in the form

$$
\begin{gathered}
u_{n}=u_{n-1}+k f^{(1)}\left(u_{n}, v_{n}, u_{n-s_{1}}, v_{n-s_{2}}\right), \\
v_{n}=v_{n-1}+k f^{(2)}\left(u_{n}, v_{n}, u_{n-s_{1}}, v_{n-s_{2}}\right) \\
\left.u_{n}=\phi_{n} \quad\left(n \in I_{1}\right), \quad v_{n}=\psi_{n} \quad\left(n \in I_{2}\right), \ldots\right),
\end{gathered}
$$

where $f^{(1)}$ and $f^{(2)}$ are, in general, nonlinear functions of their respective arguments, $k$ is a positive constant, $s_{1}$ and $s_{2}$ are positive integers, and $I_{1}$ and $I_{2}$ are subsets of nonpositive 
integers given by

$$
I_{1} \equiv\left\{-s_{1},-s_{1}+1, \ldots, 0\right\}, \quad I_{2} \equiv\left\{-s_{2},-s_{2}+1, \ldots, 0\right\} .
$$

System (1.1) is a backward (or left-sided) difference approximation of the delay differential system

$$
\begin{gathered}
\frac{d u}{d t}=f^{(1)}\left(u, v, u_{\tau_{1}}, v_{\tau_{2}}\right), \quad \frac{d v}{d t}=f^{(2)}\left(u, v, u_{\tau_{1}}, v_{\tau_{2}}\right) \quad(t>0), \\
u(t)=\phi(t) \quad\left(-\tau_{1} \leq t \leq 0\right), \quad v(t)=\psi(t) \quad\left(-\tau_{2} \leq t \leq 0\right),
\end{gathered}
$$

where $u_{\tau_{1}}=u\left(t-\tau_{1}\right), v_{\tau_{2}}=v\left(t-\tau_{2}\right)$, and $\tau_{1}$ and $\tau_{2}$ are positive constants representing the time delays. In relation to the above differential system, the constant $k$ in (1.1) plays the role of the time increment $\Delta t$ in the difference approximation and is chosen such that $s_{1} \equiv \tau_{1} / k$ and $s_{2} \equiv \tau_{2} / k$ are positive integers.

Our consideration of the difference system (1.1) is motivated by some Lotka-Volterra models in population dynamics where the effect of time delays in the opposing species is taken into consideration. The equations for the difference approximations of these model problems, referred to as cooperative, competition, and prey-predator, respectively, involve three distinct quasimonotone reaction functions, and are given as follows (cf. $[7,11,12,15,20])$ :

(a) the cooperative system:

$$
\begin{gathered}
u_{n}=u_{n-1}+k \alpha^{(1)} u_{n}\left(1-a^{(1)} u_{n}+b^{(1)} v_{n}+c^{(1)} v_{n-s_{2}}\right) \\
v_{n}=v_{n-1}+k \alpha^{(2)} v_{n}\left(1+a^{(2)} u_{n}-b^{(2)} v_{n}+c^{(2)} u_{n-s_{1}}\right) \quad(n=1,2, \ldots), \\
u_{n}=\phi_{n} \quad\left(n \in I_{1}\right), \quad v_{n}=\psi_{n} \quad\left(n \in I_{2}\right) ;
\end{gathered}
$$

(b) the competition system:

$$
\begin{gathered}
u_{n}=u_{n-1}+k \alpha^{(1)}\left(1-a^{(1)} u_{n}-b^{(1)} v_{n}-c^{(1)} v_{n-s_{2}}\right) \\
v_{n}=v_{n-1}+k \alpha^{(2)}\left(1-a^{(2)} u_{n}-b^{(2)} v_{n}-c^{(2)} u_{n-s_{1}}\right) \quad(n=1,2, \ldots), \\
u_{n}=\phi_{n} \quad\left(n \in I_{1}\right), \quad v_{n}=\psi_{n} \quad\left(n \in I_{2}\right) ;
\end{gathered}
$$

(c) the prey-predator system:

$$
\begin{gathered}
u_{n}=u_{n-1}+k \alpha^{(1)}\left(1-a^{(1)} u_{n}-b^{(1)} v_{n}-c^{(1)} v_{n-s_{2}}\right) \\
v_{n}=v_{n-1}+k \alpha^{(2)}\left(1+a^{(2)} u_{n}-b^{(2)} v_{n}+c^{(2)} u_{n-s_{1}}\right) \quad(n=1,2, \ldots), \\
u_{n}=\phi_{n} \quad\left(n \in I_{1}\right), \quad v_{n}=\psi_{n} \quad\left(n \in I_{2}\right) .
\end{gathered}
$$

In the systems (1.4), (1.5), and (1.6), $u_{n}$ and $v_{n}$ represent the densities of the two population species at time $n k(\equiv n \Delta t), k$ is a small time increment, and for each $l=1,2, \alpha^{(l)}, a^{(l)}$, $b^{(l)}$, and $c^{(l)}$ are positive constants representing the various reaction rates.

There are huge amounts of works in the literature that dealt with the asymptotic behavior of solutions for differential and difference systems with time delays, and much of 
the discussions in the earlier work are devoted to differential systems, including various Lotka-Volterra-type equations (cf. [2, 3, 5, 7, 8, 12, 15, 19, 20]). Later development leads to various forms of difference equations, and many of them are discrete analogues of differential equations (cf. $[2,3,4,5,6,8,9,10,11,19]$ ). In recent years, attention has also been given to finite-difference equations which are discrete approximations of differential equations with the effect of diffusion (cf. $[14,15,16,17,18]$ ). In this paper, we consider the coupled difference system (1.1) for a general class of reaction functions $\left(f^{(1)}, f^{(2)}\right)$, and our aim is to show the existence and uniqueness of a global positive solution and the asymptotic behavior of the solution with particular emphasis on the global attraction of a positive equilibrium solution. The results for the general system are then applied to each of the three Lotka-Volterra models in (1.4)-(1.6) where some easily verifiable conditions on the rate constants $a^{(l)}, b^{(l)}$, and $c^{(l)}, l=1,2$, are obtained so that a unique positive equilibrium solution exists and is a global attractor of the system.

The plan of the paper is as follows. In Section 2, we show the existence and uniqueness of a positive global solution to the general system (1.1) for arbitrary Lipschitz continuous functions $\left(f^{(1)}, f^{(2)}\right)$. Section 3 is concerned with some comparison theorems among solutions of (1.1) for three different types of quasimonotone functions. The asymptotic behavior of the solution is treated in Section 4 where sufficient conditions are obtained for ensuring the global attraction of a positive equilibrium solution. This global attraction property is then applied in Section 5 to the Lotka-Volterra models in (1.4), (1.5), and (1.6) which correspond to the three types of quasimonotone functions in the general system.

\section{Existence and uniqueness of positive solution}

Before discussing the asymptotic behavior of the solution of (1.1) we show the existence and uniqueness of a positive solution under the following basic hypothesis on the function $\left(f^{(1)}, f^{(2)}\right) \equiv\left(f^{(1)}\left(u, v, u_{s}, v_{s}\right), f^{(2)}\left(u, v, u_{s}, v_{s}\right)\right)$.

$\left(H_{1}\right)$ (i) The function $\left(f^{(1)}, f^{(2)}\right)$ satisfies the local Lipschitz condition

$$
\begin{aligned}
& \left|f^{(l)}\left(u, v, u_{s}, v_{s}\right)-f^{(l)}\left(u^{\prime}, v^{\prime}, u_{s}^{\prime}, v_{s}^{\prime}\right)\right| \\
& \quad \leq K^{(l)}\left(\left|u-u^{\prime}\right|+\left|v-v^{\prime}\right|+\left|u_{s}-u_{s}^{\prime}\right|+\left|v_{s}-v_{s}^{\prime}\right|\right) \\
& \quad \text { for }\left(u, v, u_{s}, v_{s}\right),\left(u^{\prime}, v^{\prime}, u_{s}^{\prime}, v_{s}^{\prime}\right) \in \mathscr{S} \times \mathscr{S},(l=1,2) .
\end{aligned}
$$

(ii) There exist positive constants $\left(M^{(1)}, M^{(2)}\right),\left(\delta^{(1)}, \delta^{(2)}\right)$ with $\left(M^{(1)}, M^{(2)}\right) \geq$ $\left(\delta^{(1)}, \delta^{(2)}\right)$ such that for all $\left(u_{s}, v_{s}\right) \in \mathscr{Y}$,

$$
\begin{array}{ll}
f^{(1)}\left(M^{(1)}, v, u_{s}, v_{s}\right) \leq 0 \leq f^{(1)}\left(\delta^{(1)}, v, u_{s}, v_{s}\right) & \text { when } \delta^{(2)} \leq v \leq M^{(2)} \\
f^{(2)}\left(u, M^{(2)}, u_{s}, v_{s}\right) \leq 0 \leq f^{(2)}\left(u, \delta^{(2)}, u_{s}, v_{s}\right) & \text { when } \delta^{(1)} \leq u \leq M^{(1)}
\end{array}
$$

In the above hypothesis, $\mathscr{Y}$ is given by

$$
\mathscr{S} \equiv\left\{(u, v) \in \mathbb{R}^{2} ;\left(\delta^{(1)}, \delta^{(2)}\right) \leq(u, v) \leq\left(M^{(1)}, M^{(2)}\right)\right\}
$$


To ensure the uniqueness of the solution, we assume that the time increment $k$ satisfies the condition

$$
k\left(K^{(1)}+K^{(2)}\right)<1,
$$

where $K^{(1)}$ and $K^{(2)}$ are the Lipschitz constants in (2.1).

Theorem 2.1. Let hypothesis $\left(H_{1}\right)$ hold. Then system (1.1) has at least one global solution $\left(u_{n}, v_{n}\right)$ in $\mathscr{Y}$. If, in addition, condition (2.4) is satisfied, then the solution $\left(u_{n}, v_{n}\right)$ is unique in $\mathscr{S}$.

Proof. Given any $W_{n} \equiv\left(w_{n}, z_{n}\right) \in \mathscr{Y}$, we let $U_{n} \equiv\left(u_{n}, v_{n}\right)$ be the solution of the uncoupled initial value problem

$$
\begin{gathered}
\left(1+k K^{(1)}\right) u_{n}=u_{n-1}+k\left[K^{(1)} w_{n}+f^{(1)}\left(w_{n}, z_{n}, u_{n-s_{1}}, v_{n-s_{2}}\right)\right], \\
\left(1+k K^{(2)}\right) v_{n}=v_{n-1}+k\left[K^{(2)} z_{n}+f^{(2)}\left(w_{n}, z_{n}, u_{n-s_{1}}, v_{n-s_{2}}\right)\right] \quad(n=1,2, \ldots), \\
u_{n}=\phi_{n} \quad\left(n \in I_{1}\right), \quad v_{n}=\psi_{n} \quad\left(n \in I_{2}\right),
\end{gathered}
$$

where $K^{(1)}$ and $K^{(2)}$ are the Lipschitz constants in (2.1). Define a solution operator $\mathscr{P}$ : $\mathscr{S} \rightarrow \mathbb{R}^{2}$ by

$$
\mathscr{P} W_{n} \equiv\left(P^{(1)} W_{n}, P^{(2)} W_{n}\right) \equiv\left(u_{n}, v_{n}\right) \quad\left(W_{n} \in \mathscr{S}\right) .
$$

Then system (1.1) may be expressed as

$$
U_{n}=\mathscr{P} U_{n}, \quad U_{n}=\left(u_{n}, v_{n}\right) \quad(n=1,2, \ldots) .
$$

To prove the existence of a global solution to (1.1) it suffices to show that $\mathscr{P}$ has a fixed point in $\mathscr{S}$ for every $n$. It is clear from hypothesis $\left(H_{1}\right)$ that $\mathscr{P}$ is a continuous map on $\mathscr{S}$ which is a closed bounded convex subset of $\mathbb{R}^{2}$. We show that $\mathscr{P}$ maps $\mathscr{Y}$ into itself by a marching process.

Given any $W_{n} \equiv\left(w_{n}, z_{n}\right) \in \mathscr{Y}$, relation (2.6) and conditions (2.1), (2.2) imply that

$$
\begin{gathered}
\left(1+k K^{(1)}\right)\left(M^{(1)}-P^{(1)} W_{n}\right) \\
=\left(1+k K^{(1)}\right) M^{(1)}-\left[u_{n-1}+k\left(K^{(1)} w_{n}+f^{(1)}\left(w_{n}, z_{n}, u_{n-s_{1}}, v_{n-s_{2}}\right)\right)\right] \\
\geq\left(M^{(1)}-u_{n-1}\right)+k\left[K^{(1)}\left(M^{(1)}-w_{n}\right)+f^{(1)}\left(M^{(1)}, z_{n}, u_{n-s_{1}}, v_{n-s_{2}}\right)\right. \\
\left.\quad-f^{(1)}\left(w_{n}, z_{n}, u_{n-s_{1}}, v_{n-s_{2}}\right)\right] \geq M^{(1)}-u_{n-1}, \\
\left(1+k K^{(2)}\right)\left(M^{(2)}-P^{(2)} W_{n}\right) \\
=\left(1+k K^{(2)}\right) M^{(2)}-\left[v_{n-1}+k\left(K^{(2)} z_{n}+f^{(2)}\left(w_{n}, z_{n}, u_{n-s_{1}}, v_{n-s_{2}}\right)\right)\right] \\
\geq\left(M^{(2)}-v_{n-1}\right)+k\left[K^{(2)}\left(M^{(2)}-z_{n}\right)+f^{(2)}\left(w_{n}, M^{(2)}, u_{n-s_{1}}, v_{n-s_{2}}\right)\right. \\
\left.\quad-f^{(2)}\left(w_{n}, z_{n}, u_{n-s_{1}}, v_{n-s_{2}}\right)\right] \\
\geq M^{(2)}-v_{n-1} \quad(n=1,2, \ldots),
\end{gathered}
$$


whenever $\left(u_{n-s_{1}}, v_{n-s_{2}}\right) \in \mathscr{Y}$. This leads to the relation

$$
\begin{gathered}
M^{(1)}-P^{(1)} W_{n} \geq \frac{M^{(1)}-u_{n-1}}{1+k K^{(1)}} \\
M^{(2)}-P^{(2)} W_{n} \geq \frac{M^{(2)}-v_{n-1}}{1+k K^{(2)}} \quad(n=1,2, \ldots) .
\end{gathered}
$$

A similar argument using the second inequalities in (2.2) gives

$$
\begin{gathered}
P^{(1)} W_{n}-\delta^{(1)} \geq \frac{u_{n-1}-\delta^{(1)}}{1+k K^{(1)}}, \\
P^{(2)} W_{n}-\delta^{(2)} \geq \frac{v_{n-1}-\delta^{(2)}}{1+k K^{(2)}} \quad(n=1,2, \ldots),
\end{gathered}
$$

whenever $\left(u_{n-s_{1}}, v_{n-s_{2}}\right) \in \mathscr{S}$. Consider the case $n=1$. Since $\left(u_{1-s_{1}}, v_{1-s_{2}}\right)=\left(\phi_{1-s_{1}}, \psi_{1-s_{2}}\right)$ and $\left(u_{0}, v_{0}\right)=\left(\phi_{0}, \psi_{0}\right)$ are in $\mathscr{S}$, relations (2.9), (2.10) imply that $\left(\delta^{(1)}, \delta^{(2)}\right) \leq\left(P^{(1)} W_{1}\right.$, $\left.P^{(2)} W_{1}\right) \leq\left(M^{(1)}, M^{(2)}\right)$. By Brower's fixed point theorem, $\mathscr{P} \equiv\left(P^{(1)}, P^{(2)}\right)$ has a fixed point $U_{1} \equiv\left(u_{1}, v_{1}\right)$ in $\mathscr{Y}$. This shows that $\left(u_{1}, v_{1}\right)$ is a solution of $(1.1)$ for $n=1$, and $\left(u_{1}, v_{1}\right)$ and $\left(u_{2-s_{1}}, v_{2-s_{2}}\right)$ are in $\mathscr{Y}$. Using this property in (2.9), (2.10) for $n=2$, the same argument shows that $\mathscr{P}$ has a fixed point $U_{2} \equiv\left(u_{2}, v_{2}\right)$ in $\mathscr{S}$, and $\left(u_{2}, v_{2}\right)$ is a solution of $(1.1)$ for $n=2$ and $\left(u_{3-s_{1}}, v_{3-s_{2}}\right) \in \mathscr{Y}$. A continuation of the above argument shows that $\mathscr{P}$ has a fixed point $U_{n} \equiv\left(u_{n}, v_{n}\right)$ in $\mathscr{Y}$ for every $n$, and $\left(u_{n}, v_{n}\right)$ is a global solution of (1.1) in $\mathscr{Y}$.

To show the uniqueness of the solution, we consider any two solutions $\left(u_{n}, v_{n}\right),\left(u_{n}^{\prime}, v_{n}^{\prime}\right)$ in $\mathscr{S}$ and let $\left(\bar{w}_{n}, \bar{z}_{n}\right)=\left(u_{n}-u_{n}^{\prime}, v_{n}-v_{n}^{\prime}\right)$. By $(1.1)$,

$$
\begin{gathered}
\bar{w}_{n}=\bar{w}_{n-1}+k\left[f^{(1)}\left(u_{n}, v_{n}, u_{n-s_{1}}, v_{n-s_{2}}\right)-f^{(1)}\left(u_{n}^{\prime}, v_{n}^{\prime}, u_{n-s_{1}}^{\prime}, v_{n-s_{2}}^{\prime}\right)\right], \\
\bar{z}_{n}=\bar{z}_{n-1}+k\left[f^{(2)}\left(u_{n}, v_{n}, u_{n-s_{1}}, v_{n-s_{2}}\right)-f^{(2)}\left(u_{n}^{\prime}, v_{n}^{\prime}, u_{n-s_{1}}^{\prime}, v_{n-s_{2}}^{\prime}\right)\right] \quad(n=1,2, \ldots), \\
\bar{w}_{n}=0 \quad\left(n \in I_{1}\right), \quad \bar{z}_{n}=0 \quad\left(n \in I_{2}\right) .
\end{gathered}
$$

The above relation and condition (2.1) imply that

$$
\begin{aligned}
\left|\bar{w}_{n}\right| & \leq\left|\bar{w}_{n-1}\right|+k K^{(1)}\left(\left|\bar{w}_{n}\right|+\left|\bar{z}_{n}\right|+\left|\bar{w}_{n-s_{1}}\right|+\left|\bar{z}_{n-s_{2}}\right|\right), \\
\left|\bar{z}_{n}\right| & \leq\left|\bar{z}_{n-1}\right|+k K^{(2)}\left(\left|\bar{w}_{n}\right|+\left|\bar{z}_{n}\right|+\left|\bar{w}_{n-s_{1}}\right|+\left|\bar{z}_{n-s_{2}}\right|\right) .
\end{aligned}
$$

Addition of the above inequalities leads to

$$
\begin{aligned}
\left|\bar{w}_{n}\right|+\left|\bar{z}_{n}\right| \leq & \left|\bar{w}_{n-1}\right|+\left|\bar{z}_{n-1}\right| \\
& +k\left(K^{(1)}+K^{(2)}\right)\left(\left|\bar{w}_{n}\right|+\left|\bar{z}_{n}\right|+\left|\bar{w}_{n-s_{1}}\right|+\left|\bar{z}_{n-s_{2}}\right|\right) \quad(n=1,2, \ldots) .
\end{aligned}
$$

Since $\bar{w}_{n}=\bar{z}_{n}=0$ for $n=0,-1,-2, \ldots$, the above inequality for $n=1$ yields

$$
\left|\bar{w}_{1}\right|+\left|\bar{z}_{1}\right| \leq k\left(K^{(1)}+K^{(2)}\right)\left(\left|\bar{w}_{1}\right|+\left|\bar{z}_{1}\right|\right) .
$$

In view of condition (2.4), this is possible only when $\left|\bar{w}_{1}\right|=\left|\bar{z}_{1}\right|=0$. Using $\bar{w}_{1}=\bar{z}_{1}=0$ in (2.13) for $n=2$ yields

$$
\left|\bar{w}_{2}\right|+\left|\bar{z}_{2}\right| \leq k\left(K^{(1)}+K^{(2)}\right)\left(\left|\bar{w}_{2}\right|+\left|\bar{z}_{2}\right|\right) .
$$


It follows again from (2.4) that $\left|\bar{w}_{2}\right|=\left|\bar{z}_{2}\right|=0$. The conclusion $\left|\bar{w}_{n}\right|=\left|\bar{z}_{n}\right|=0$ for every $n$ follows by an induction argument. This proves $\left(u_{n}, v_{n}\right)=\left(u_{n}^{\prime}, v_{n}^{\prime}\right)$, and therefore $\left(u_{n}, v_{n}\right)$ is the unique solution of (1.1) in $\mathscr{Y}$.

Remark 2.2. (a) Since problem (1.3) may be considered as an equivalent system of the scalar second-order differential equation

$$
\begin{gathered}
u^{\prime \prime}=f\left(u, u^{\prime}, u_{\tau_{1}}, u_{\tau_{2}}^{\prime}\right) \quad(t>0), \\
u(t)=\phi(t) \quad\left(-\tau_{1} \leq t \leq 0\right), \quad u^{\prime}(t)=\psi(t) \quad\left(-\tau_{2} \leq t \leq 0\right),
\end{gathered}
$$

the conclusion in Theorem 2.1 and all the results obtained in later sections are directly applicable to the difference approximation of (2.16) with $\left(u_{n}, v_{n}\right)=\left(u_{n}, u_{n}^{\prime}\right)$ and $\left(f^{(1)}\right.$, $\left.f^{(2)}\right)=\left(v_{n}, f\left(u_{n}, v_{n}, u_{n-s_{1}}, v_{n-s_{2}}\right)\right)$.

(b) System (1.1) is a difference approximation of (1.3) by the backward (or left-sided) approximation of the time derivative $(d u / d t, d v / d t)$, and this approximation preserves the nonlinear nature of the differential system. If the forward (or right-sided) approximation for $(d u / d t, d v / d t)$ is used, then the resulting difference system gives an explicit formula for $\left(u_{n+1}, v_{n+1}\right)$ which can be computed by a marching process for every $n=0,1,2, \ldots$ and for any continuous function $\left(f^{(1)}, f^{(2)}\right)$. From a view point of differential equations, the forward approximation may lead to misleading information about the solution of the differential system. One reason is that a global solution to the differential system may fail to exist while the difference solution $\left(u_{n+1}, v_{n+1}\right)$ exists for every $n$.

(c) The uniqueness result in Theorem 2.1 is in the set $\mathscr{Y}$, and it does not rule out the possibility of existence of positive solutions outside of $\mathscr{Y}$.

\section{Comparison theorems}

To investigate the asymptotic behavior of the solution we consider a class of quasimonotone functions which depend on the monotone property of $\left(f^{(1)}, f^{(2)}\right)$. Specifically, we make the following hypothesis.

$\left(H_{2}\right)\left(f^{(1)}, f^{(2)}\right)$ is a $C^{1}$-function in $\mathscr{S} \times \mathscr{S}$ and possesses the property $\partial f^{(1)} / \partial u_{s} \geq 0$, $\partial f^{(2)} / \partial v_{s} \geq 0$ and one of the following quasimonotone properties for $\left(u, v, u_{s}, v_{s}\right) \in$ $\mathscr{S} \times \mathscr{S}:$

(a) quasimonotone nondecreasing:

$$
\frac{\partial f^{(1)}}{\partial v} \geq 0, \quad \frac{\partial f^{(1)}}{\partial v_{s}} \geq 0, \quad \frac{\partial f^{(2)}}{\partial u} \geq 0, \quad \frac{\partial f^{(2)}}{\partial u_{s}} \geq 0
$$

(b) quasimonotone nonincreasing:

$$
\frac{\partial f^{(1)}}{\partial v} \leq 0, \quad \frac{\partial f^{(1)}}{\partial v_{s}} \leq 0, \quad \frac{\partial f^{(2)}}{\partial u} \leq 0, \quad \frac{\partial f^{(2)}}{\partial u_{s}} \leq 0 ;
$$

(c) mixed quasimonotone:

$$
\frac{\partial f^{(1)}}{\partial v} \leq 0, \quad \frac{\partial f^{(1)}}{\partial v_{s}} \leq 0, \quad \frac{\partial f^{(2)}}{\partial u} \geq 0, \quad \frac{\partial f^{(2)}}{\partial u_{s}} \geq 0 .
$$


Notice that if $\left(f^{(1)}, f^{(2)}\right) \equiv\left(f^{(1)}(u, v), f^{(2)}(u, v)\right)$ is independent of $\left(u_{s}, v_{s}\right)$, then the above conditions are reduced to those required for the standard three types of quasimonotone functions (cf. $[15,18])$.

It is easy to see from $\left(H_{2}\right)$ that for quasimonotone functions the conditions on $\left(M^{(1)}\right.$, $\left.M^{(2)}\right),\left(\delta^{(1)}, \delta^{(2)}\right)$ in $(2.2)$ are reduced to the following.

(a) For quasimonotone nondecreasing functions:

$$
\begin{aligned}
& f^{(1)}\left(M^{(1)}, M^{(2)}, M^{(1)}, M^{(2)}\right) \leq 0 \leq f^{(1)}\left(\delta^{(1)}, \delta^{(2)}, \delta^{(1)}, \delta^{(2)}\right), \\
& f^{(2)}\left(M^{(1)}, M^{(2)}, M^{(1)}, M^{(2)}\right) \leq 0 \leq f^{(2)}\left(\delta^{(1)}, \delta^{(2)}, \delta^{(1)}, \delta^{(2)}\right) .
\end{aligned}
$$

(b) For quasimonotone nonincreasing functions:

$$
\begin{aligned}
& f^{(1)}\left(M^{(1)}, \delta^{(2)}, M^{(1)}, \delta^{(2)}\right) \leq 0 \leq f^{(1)}\left(\delta^{(1)}, M^{(2)}, \delta^{(1)}, M^{(2)}\right), \\
& f^{(2)}\left(\delta^{(1)}, M^{(2)}, \delta^{(1)}, M^{(2)}\right) \leq 0 \leq f^{(2)}\left(M^{(1)}, \delta^{(2)}, M^{(1)}, \delta^{(2)}\right) .
\end{aligned}
$$

(c) For mixed quasimonotone functions:

$$
\begin{aligned}
& f^{(1)}\left(M^{(1)}, \delta^{(2)}, M^{(1)}, \delta^{(2)}\right) \leq 0 \leq f^{(1)}\left(\delta^{(1)}, M^{(2)}, \delta^{(1)}, M^{(2)}\right), \\
& f^{(2)}\left(M^{(1)}, M^{(2)}, M^{(1)}, M^{(2)}\right) \leq 0 \leq f^{(2)}\left(\delta^{(1)}, \delta^{(2)}, \delta^{(1)}, \delta^{(2)}\right) .
\end{aligned}
$$

In this section, we show some comparison results among solutions with different initial functions for each of the above three types of quasimonotone functions. The comparison results for the first two types of quasimonotone functions are based on the following positivity lemma for a function $\left(w_{n}, z_{n}\right)$ satisfying the relation

$$
\begin{gathered}
\gamma_{n}^{(1)} w_{n} \geq w_{n-1}+a_{n}^{(1)} z_{n}+b_{n}^{(1)} w_{n-s_{1}}+c_{n}^{(1)} z_{n-s_{2}}, \\
\gamma_{n}^{(2)} z_{n} \geq z_{n-1}+a_{n}^{(2)} w_{n}+b_{n}^{(2)} w_{n-s_{1}}+c_{n}^{(2)} z_{n-s_{2}} \quad(n=1,2, \ldots), \\
w_{n} \geq 0 \quad\left(n \in I_{1}\right), \quad z_{n} \geq 0 \quad(n \in I),
\end{gathered}
$$

where for each $l=1,2$, and $n=1,2, \ldots, \gamma_{n}^{(l)}$ is positive, and $a_{n}^{(l)}, b_{n}^{(l)}$, and $c_{n}^{(l)}$ are nonnegative.

Lemma 3.1. Let $\left(w_{n}, z_{n}\right)$ satisfy (3.7), and let

$$
a_{n}^{(1)} a_{n}^{(2)}<\gamma_{n}^{(1)} \gamma_{n}^{(2)} \quad(n=1,2, \ldots)
$$

Then $\left(w_{n}, z_{n}\right) \geq(0,0)$ for every $n=1,2, \ldots$.

Proof. Consider the case $n=1$. Since $w_{n} \geq 0$ for $n \in I_{1}$ and $z_{n} \geq 0$ for $n \in I_{2}$, the inequalities in (3.7) yield

$$
\gamma_{1}^{(1)} w_{1} \geq a_{1}^{(1)} z_{1}, \quad \gamma_{1}^{(2)} z_{1} \geq a_{1}^{(2)} w_{1}
$$


The positivity of $\gamma_{1}^{(1)}, \gamma_{1}^{(2)}$ implies that

$$
\begin{aligned}
& w_{1} \geq\left(\frac{a_{1}^{(1)}}{\gamma_{1}^{(1)}}\right) z_{1} \geq\left(\frac{a_{1}^{(1)} a_{1}^{(2)}}{\gamma_{1}^{(1)} \gamma_{1}^{(2)}}\right) w_{1}, \\
& z_{1} \geq\left(\frac{a_{1}^{(2)}}{\gamma_{1}^{(2)}}\right) w_{1} \geq\left(\frac{a_{1}^{(1)} a_{1}^{(2)}}{\gamma_{1}^{(1)} \gamma_{1}^{(2)}}\right) z_{1} .
\end{aligned}
$$

In view of (3.8), the above inequalities can hold only if $\left(w_{1}, z_{1}\right) \geq(0,0)$. Assume, by induction, that $\left(w_{n}, z_{n}\right) \geq(0,0)$ for $n=1,2, \ldots, m-1$ for some $m>1$. Then by (3.7),

$$
\gamma_{m}^{(1)} w_{m} \geq a_{m}^{(1)} z_{m}, \quad \gamma_{m}^{(2)} z_{m} \geq a_{m}^{(2)} w_{m}
$$

This leads to

$$
w_{m} \geq\left(\frac{a_{m}^{(1)} a_{m}^{(2)}}{\gamma_{m}^{(1)} \gamma_{m}^{(2)}}\right), \quad z_{m} \geq\left(\frac{a_{m}^{(1)} a_{m}^{(2)}}{\gamma_{m}^{(1)} \gamma_{m}^{(2)}}\right) z_{m} .
$$

It follows again from $(3.8)$ that $\left(w_{m}, z_{m}\right) \geq(0,0)$. The conclusion of the lemma follows by the principle of induction.

The above positivity lemma can be extended to a function $\left(\bar{w}_{n}, \bar{z}_{n}, \underline{w}_{n}, \underline{z}_{n}\right)$ satisfying the relation

$$
\begin{gathered}
\gamma_{n}^{(1)} \bar{w}_{n} \geq \bar{w}_{n-1}+a_{n}^{(1)} \underline{z}_{n}+b_{n}^{(1)} \bar{w}_{n-s_{1}}+c_{n}^{(1)} \underline{z}_{n-s_{2}}, \\
\gamma_{n}^{(2)} \bar{z}_{n} \geq \bar{z}_{n-1}+a_{n}^{(2)} \bar{w}_{n}+b_{n}^{(2)} \bar{w}_{n-s_{1}}+c_{n}^{(2)} \bar{z}_{n-s_{2}}, \\
\hat{\gamma}_{n}^{(1)} \underline{w}_{n} \geq \underline{w}_{n-1}+\hat{a}_{n}^{(1)} \bar{z}_{n}+\hat{b}_{n}^{(1)} \underline{w}_{n-s_{1}}+\hat{c}_{n}^{(1)} \bar{z}_{n-s_{2}}, \\
\hat{\gamma}_{n}^{(2)} \underline{z}_{n} \geq \underline{z}_{n-1}+\hat{a}_{n}^{(2)} \underline{w}_{n}+\hat{b}_{n}^{(2)} \underline{w}_{n-s_{1}}+\hat{c}_{n}^{(2)} \underline{z}_{n-s_{2}} \quad(n=1,2, \ldots), \\
\bar{w}_{n} \geq 0, \quad \underline{w}_{n} \geq 0 \quad\left(n \in I_{1}\right), \quad \bar{z}_{n} \geq 0, \quad \underline{z}_{n} \geq 0 \quad\left(n \in I_{2}\right),
\end{gathered}
$$

where $\gamma_{n}^{(l)}, a_{n}^{(l)}, b_{n}^{(l)}$, and $c_{n}^{(l)}, l=1,2$, are the same as that in $(3.7)$ and $\hat{\gamma}_{n}^{(l)}, \hat{a}_{n}^{(l)}, \hat{b}_{n}^{(l)}$, and $\hat{c}_{n}^{(l)}$ are nonnegative with $\hat{\gamma}_{n}^{(l)}>0, n=1,2, \ldots$

LEMma 3.2. Let $\left(\bar{w}_{n}, \bar{z}_{n}, \underline{w}_{n}, \underline{z}_{n}\right)$ satisfy (3.13), and let

$$
\left(a_{n}^{(1)} a_{n}^{(2)}\right)\left(\hat{a}_{n}^{(1)} \hat{a}_{n}^{(2)}\right)<\left(\gamma_{n}^{(1)} \gamma_{n}^{(2)}\right)\left(\hat{\gamma}_{n}^{(1)} \hat{\gamma}_{n}^{(2)}\right) \quad(n=1,2, \ldots)
$$

Then $\left(\bar{w}_{n}, \bar{z}_{n}, \underline{w}_{n}, \underline{z}_{n}\right) \geq(0,0,0,0)$ for every $n$.

Proof. By (3.13) with $n=1$, we have

$$
\gamma_{1}^{(1)} \bar{w}_{1} \geq a_{1}^{(1)} \underline{z}_{1}, \quad \gamma_{1}^{(2)} \bar{z}_{1} \geq a_{1}^{(2)} \bar{w}_{1}, \quad \hat{\gamma}_{1}^{(1)} \underline{w}_{1} \geq \hat{a}_{1}^{(1)} \bar{z}_{1}, \quad \hat{\gamma}_{1}^{(2)} \underline{z}_{1} \geq \hat{a}_{1}^{(2)} \underline{w}_{1} .
$$


This implies that

$$
\begin{aligned}
\bar{w}_{1} & \geq\left(\frac{a_{1}^{(1)}}{\gamma_{1}^{(1)}}\right) \underline{z}_{1} \geq\left(\frac{a_{1}^{(1)}}{\gamma_{1}^{(1)}}\right)\left(\frac{\hat{a}_{1}^{(2)}}{\hat{\gamma}_{1}^{(2)}}\right) \underline{w}_{1} \geq\left(\frac{a_{1}^{(1)}}{\gamma_{1}^{(1)}}\right)\left(\frac{\hat{a}_{1}^{(2)}}{\hat{\gamma}_{1}^{(2)}}\right)\left(\frac{\hat{a}_{1}^{(1)}}{\hat{\gamma}_{1}^{(1)}}\right) \bar{z}_{1} \\
& \geq\left(\frac{a_{1}^{(1)} a_{1}^{(2)}}{\gamma_{1}^{(1)} \gamma_{2}^{(2)}}\right)\left(\frac{\hat{a}_{1}^{(1)} \hat{a}_{2}^{(2)}}{\hat{\gamma}_{1}^{(1)} \hat{\gamma}_{1}^{(2)}}\right) \bar{w}_{1} .
\end{aligned}
$$

In view of condition (3.14), we have $\bar{w}_{1} \geq 0$. This implies that $\bar{z}_{1} \geq 0, \underline{w}_{1} \geq 0$ and $\underline{z}_{1} \geq 0$ which proves the case for $n=1$. Assume, by induction, that $\left(\bar{w}_{n}, \bar{z}_{n}, \underline{w}_{n}, \underline{z}_{n}\right) \geq(0,0,0,0)$ for $n=1,2, \ldots, m-1$ for some $m>1$. Then by (3.13),

$$
\gamma_{m}^{(1)} \bar{w}_{m} \geq a_{m}^{(1)} \underline{z}_{m}, \quad \gamma_{m}^{(2)} \bar{z}_{m} \geq a_{m}^{(2)} \bar{w}_{m}, \quad \hat{\gamma}_{m}^{(1)} \underline{w}_{m} \geq \hat{a}_{m}^{(1)} \bar{z}_{m}, \quad \hat{\gamma}_{m}^{(2)} \underline{z}_{m} \geq \hat{a}_{m}^{(2)} \underline{w}_{m} .
$$

This leads to

$$
\bar{w}_{m} \geq\left(\frac{a_{m}^{(1)} a_{m}^{(2)}}{\gamma_{m}^{(1)} \gamma_{m}^{(2)}}\right)\left(\frac{\hat{a}_{m}^{(1)} \hat{a}_{m}^{(2)}}{\hat{\gamma}_{m}^{(1)} \hat{\gamma}_{m}^{(2)}}\right) \bar{w}_{m} .
$$

It follows again from (3.14) that $\bar{w}_{m} \geq 0$ from which we obtain $\bar{z}_{m} \geq 0, \underline{w}_{m} \geq 0$ and $\underline{z}_{m} \geq 0$. The conclusion of the lemma follows from the principle of induction.

To obtain comparison results among solutions, we need to impose a condition on the time increment $k$. Define

$$
\begin{gathered}
\sigma_{1}^{(1)} \equiv \max \left\{\frac{\partial f^{(1)}}{\partial u}\left(u, v, u_{s}, v_{s}\right) ;(u, v),\left(u_{s}, v_{s}\right) \in \mathscr{S}\right\}, \\
\sigma_{2}^{(1)} \equiv \max \left\{\left|\frac{\partial f^{(1)}}{\partial v}\left(u, v, u_{s}, v_{s}\right)\right| ;(u, v),\left(u_{s}, v_{s}\right) \in \mathscr{S}\right\}, \\
\sigma_{1}^{(2)} \equiv \max \left\{\left|\frac{\partial f^{(2)}}{\partial u}\left(u, v, u_{s}, v_{s}\right)\right| ;(u, v),\left(u_{s}, v_{s}\right) \in \mathscr{Y}\right\}, \\
\sigma_{2}^{(2)} \equiv \max \left\{\frac{\partial f^{(2)}}{\partial v}\left(u, v, u_{s}, v_{s}\right) ;(u, v),\left(u_{s}, v_{s}\right) \in \mathscr{S}\right\} .
\end{gathered}
$$

Our condition on $k$ is given by

$$
k\left(K^{(1)}+K^{(2)}\right)<1, \quad\left(k \sigma_{2}^{(1)}\right)\left(k \sigma_{1}^{(2)}\right)<\left(1-k \sigma_{1}^{(1)}\right)\left(1-k \sigma_{2}^{(2)}\right),
$$

where $K^{(l)}, l=1,2$, are the Lipschitz constants in (2.1). Since $\sigma_{1}^{(1)} \leq K^{(1)}, \sigma_{2}^{(2)} \leq K^{(2)}$, it follows that $k \sigma_{1}^{(1)}<1$ and $k \sigma_{2}^{(2)}<1$. Notice that $\sigma_{2}^{(1)}$ and $\sigma_{1}^{(2)}$ are nonnegative while $\sigma_{1}^{(1)}$ and $\sigma_{2}^{(2)}$ are not necessarily nonnegative. The following comparison theorem is for quasimonotone nondecreasing functions. 
Theorem 3.3. Let hypotheses $\left(H_{1}\right),\left(H_{2}\right)(a)$, and condition (3.20) be satisfied. Denote by $\left(\bar{u}_{n}, \bar{v}_{n}\right),\left(\underline{u}_{n}, \underline{v}_{n}\right)$, and $\left(u_{n}, v_{n}\right)$ the solutions of $(1.1)$ with $\left(\phi_{n}, \psi_{n}\right)=\left(M^{(1)}, M^{(2)}\right),\left(\phi_{n}, \psi_{n}\right)=$ $\left(\delta^{(1)}, \delta^{(2)}\right)$, and arbitrary $\left(\phi_{n}, \psi_{n}\right) \in \mathscr{Y}$, respectively. Then

$$
\left(\underline{u}_{n}, \underline{v}_{n}\right) \leq\left(u_{n}, v_{n}\right) \leq\left(\bar{u}_{n}, \bar{v}_{n}\right), \quad n=1,2, \ldots
$$

Proof. Let $\left(w_{n}, z_{n}\right)=\left(u_{n}-\underline{u}_{n}, v_{n}-\underline{v}_{n}\right)$. By (1.1) and the mean value theorem,

$$
\begin{aligned}
w_{n}=w_{n-1}+k\left[f^{(1)}\left(u_{n}, v_{n}, u_{n-s_{1}}, v_{n-s_{2}}\right)-f^{(1)}\left(\underline{u}_{n}, \underline{v}_{n}, \underline{u}_{n-s_{1}}, \underline{v}_{n-s_{2}}\right)\right] \\
=w_{n-1}+k\left[\left(\frac{\partial f^{(1)}}{\partial u}\left(\boldsymbol{\xi}_{n}\right)\right) w_{n}+\left(\frac{\partial f^{(1)}}{\partial v}\left(\boldsymbol{\xi}_{n}\right)\right) z_{n}\right. \\
\left.+\left(\frac{\partial f^{(1)}}{\partial u_{s}}\left(\boldsymbol{\xi}_{n}\right)\right) w_{n-s_{1}}+\left(\frac{\partial f^{(1)}}{\partial v_{s}}\left(\boldsymbol{\xi}_{n}\right)\right) z_{n-s_{2}}\right], \\
z_{n}=z_{n-1}+k\left[f^{(2)}\left(u_{n}, v_{n}, u_{n-s_{1}}, v_{n-s_{2}}\right)-f^{(2)}\left(\underline{u}_{n}, \underline{v}_{n}, \underline{u}_{n-s_{1}}, \underline{v}_{n-s_{2}}\right)\right] \\
=z_{n-1}+k\left[\left(\frac{\partial f^{(2)}}{\partial u}\left(\boldsymbol{\xi}_{n}^{\prime}\right)\right) w_{n}+\left(\frac{\partial f^{(2)}}{\partial v}\left(\boldsymbol{\xi}_{n}^{\prime}\right)\right) z_{n}\right. \\
\left.\quad+\left(\frac{\partial f^{(2)}}{\partial u_{s}}\left(\boldsymbol{\xi}_{n}^{\prime}\right)\right) w_{n-s_{1}}+\left(\frac{\partial f^{(2)}}{\partial v_{s}}\left(\boldsymbol{\xi}_{n}^{\prime}\right)\right) z_{n-s_{2}}\right] \quad(n=1,2, \ldots), \\
w_{n}=\phi_{n}-\delta^{(1)} \geq 0 \quad\left(n \in I_{1}\right), \quad z_{n}=\psi_{n}-\delta^{(2)} \geq 0 \quad\left(n \in I_{2}\right),
\end{aligned}
$$

where $\xi_{n} \equiv\left(\xi_{n}, \eta_{n}, \xi_{n-s_{1}}, \eta_{n-s_{2}}\right)$ and $\xi_{n}^{\prime} \equiv\left(\xi_{n}^{\prime}, \eta_{n}^{\prime}, \xi_{n-s_{1}}^{\prime}, \eta_{n-s_{2}}^{\prime}\right)$ are some intermediate values between $\left(u_{n}, v_{n}, u_{n-s_{1}}, v_{n-s_{2}}\right)$ and $\left(\underline{u}_{n}, \underline{v}_{n}, \underline{u}_{n-s_{1}}, \underline{v}_{n-s_{2}}\right)$ and therefore are in $\mathscr{Y} \times \mathscr{Y}$. Define

$$
\begin{aligned}
& \alpha_{n}^{(1)}=k \frac{\partial f^{(1)}}{\partial u}\left(\xi_{n}\right), \quad a_{n}^{(1)}=k \frac{\partial f^{(1)}}{\partial v}\left(\boldsymbol{\xi}_{n}\right), \quad b_{n}^{(1)}=k \frac{\partial f^{(1)}}{\partial u_{s}}\left(\boldsymbol{\xi}_{n}\right), \quad c_{n}^{(1)}=k \frac{\partial f^{(1)}}{\partial v_{s}}\left(\boldsymbol{\xi}_{n}\right), \\
& \alpha_{n}^{(2)}=k \frac{\partial f^{(2)}}{\partial v}\left(\boldsymbol{\xi}_{n}^{\prime}\right), \quad a_{n}^{(2)}=k \frac{\partial f^{(2)}}{\partial u}\left(\boldsymbol{\xi}_{n}^{\prime}\right), \quad b_{n}^{(2)}=k \frac{\partial f^{(2)}}{\partial u_{s}}\left(\boldsymbol{\xi}_{n}^{\prime}\right), \quad c_{n}^{(2)}=k \frac{\partial f^{(2)}}{\partial v_{s}}\left(\boldsymbol{\xi}_{n}^{\prime}\right) .
\end{aligned}
$$

Then (3.22) may be written as

$$
\begin{gathered}
\left(1-\alpha_{n}^{(1)}\right) w_{n}=w_{n-1}+a_{n}^{(1)} z_{n}+b_{n}^{(1)} w_{n-s_{1}}+c_{n}^{(1)} z_{n-s_{2}}, \\
\left(1-\alpha_{n}^{(2)}\right) z_{n}=z_{n-1}+a_{n}^{(2)} w_{n}+b_{n}^{(2)} w_{n-s_{1}}+c_{n}^{(2)} z_{n-s_{2}} \quad(n=1,2, \ldots), \\
w_{n} \geq 0 \quad\left(n \in I_{1}\right), \quad z_{n} \geq 0 \quad\left(n \in I_{2}\right) .
\end{gathered}
$$

Since by hypothesis $\left(H_{2}\right)(\mathrm{a}), a_{n}^{(l)}, b_{n}^{(l)}$, and $c_{n}^{(l)}$ are nonnegative, and by conditions (3.19) and (3.20),

$$
\begin{gathered}
\gamma_{n}^{(1)} \equiv 1-\alpha_{n}^{(1)} \geq 1-k \sigma_{1}^{(1)}>0, \quad \gamma_{n}^{(2)} \equiv 1-\alpha_{n}^{(2)} \geq 1-k \sigma_{2}^{(2)}>0, \\
a_{n}^{(1)} a_{n}^{(2)} \leq\left(k \sigma_{2}^{(1)}\right)\left(k \sigma_{1}^{(2)}\right)<\left(1-k \sigma_{1}^{(1)}\right)\left(1-k \sigma_{2}^{(2)}\right) \leq \gamma_{n}^{(1)} \gamma_{n}^{(2)} \quad(n=1,2, \ldots),
\end{gathered}
$$


we conclude from Lemma 3.1 that $\left(w_{n}, z_{n}\right) \geq(0,0)$. This leads to $\left(u_{n}, v_{n}\right) \geq\left(\underline{u}_{n}, \underline{v}_{n}\right)$. A similar argument using the property $\left(\phi_{n}, \psi_{n}\right) \leq\left(M^{(1)}, M^{(2)}\right)$ yields $\left(\bar{u}_{n}, \bar{v}_{n}\right) \geq\left(u_{n}, v_{n}\right)$. This proves the theorem.

For quasimonotone nonincreasing functions, we have the following analogous theorem.

Theorem 3.4. Let hypotheses $\left(H_{1}\right),\left(H_{2}\right)(b)$ and condition (3.20) be satisfied. Denote by $\left(\bar{u}_{n}, \underline{v}_{n}\right),\left(\underline{u}_{n}, \bar{v}_{n}\right)$, and $\left(u_{n}, v_{n}\right)$ the solutions of $(1.1)$ with $\left(\phi_{n}, \psi_{n}\right)=\left(M^{(1)}, \delta^{(2)}\right),\left(\phi_{n}, \psi_{n}\right)=$ $\left(\delta^{(1)}, M^{(2)}\right)$, and arbitrary $\left(\phi_{n}, \psi_{n}\right)$ in $\mathscr{Y}$, respectively. Then

$$
\underline{u}_{n} \leq u_{n} \leq \bar{u}_{n}, \quad \underline{v}_{n} \leq v_{n} \leq \bar{v}_{n} \quad(n=1,2, \ldots) .
$$

Proof. Consider the solutions $\left(\bar{u}_{n}, \underline{v}_{n}\right),\left(u_{n}, v_{n}\right)$ and let $w_{n}=\bar{u}_{n}-u_{n}, z_{n}=v_{n}-\underline{v}_{n}$. By (1.1) and the mean-value theorem,

$$
\begin{aligned}
w_{n} & =w_{n-1}+k\left[f^{(1)}\left(\bar{u}_{n}, \underline{v}_{n}, \bar{u}_{n-s_{1}}, \underline{v}_{n-s_{2}}\right)-f^{(1)}\left(u_{n}, v_{n}, u_{n-s_{1}}, v_{n-s_{2}}\right)\right] \\
& =w_{n-1}+\alpha_{n}^{(1)} w_{n}-a_{n}^{(1)} z_{n}+b_{n}^{(1)} w_{n-s_{1}}-c_{n}^{(1)} z_{n-s_{2}}, \\
z_{n} & =z_{n-1}+k\left[f^{(2)}\left(u_{n}, v_{n}, u_{n-s_{1}}, v_{n-s_{2}}\right)-f^{(2)}\left(\bar{u}_{n}, \underline{v}_{n}, \bar{u}_{n-s_{1}}, \underline{v}_{n-s_{2}}\right)\right] \\
& =z_{n-1}-a_{n}^{(2)} w_{n}+\alpha_{n}^{(2)} z_{n}-b_{n}^{(2)} w_{n-s_{1}}+c_{n}^{(2)} z_{n-s_{2}} \quad(n=1,2, \ldots), \\
w_{n} & =M_{n}-\phi_{n} \geq 0 \quad\left(n \in I_{1}\right), \quad z_{n}=\psi_{n}-\delta^{(2)} \geq 0 \quad\left(n \in I_{2}\right),
\end{aligned}
$$

where $\alpha_{n}^{(l)}, a_{n}^{(l)}, b_{n}^{(l)}$, and $c_{n}^{(l)}, l=1,2$, are given by (3.23) (with possibly some different intermediate values in $\mathscr{S}$ ). Since the above relation can be written in the form (3.24) with $a_{n}^{(1)}, c_{n}^{(1)}, a_{n}^{(2)}$, and $b_{n}^{(2)}$ replaced, respectively, by $\left(-a_{n}^{(1)}\right),\left(-c_{n}^{(1)}\right),\left(-a_{n}^{(2)}\right)$, and $\left(-b_{n}^{(2)}\right)$ which are nonnegative because of the quasimonotone nonincreasing property of $\left(f^{(1)}, f^{(2)}\right)$ in hypothesis $\left(H_{2}\right)(\mathrm{b})$, we conclude from the argument in the proof of Theorem 3.3 that $\left(w_{n}, z_{n}\right) \geq(0,0)$. This leads to $\bar{u}_{n} \geq u_{n}, v_{n} \geq \underline{v}_{n}$. A similar argument using $\phi_{n} \geq \delta^{(1)}, \psi_{n} \leq M^{(2)}$ gives $u_{n} \geq \underline{u}_{n}, \bar{v}_{n} \geq v_{n}$. This proves the theorem.

For mixed quasimonotone functions, we consider the solution $\left(\left(\bar{u}_{n}, \bar{v}_{n}\right),\left(\underline{u}_{n}, \underline{v}_{n}\right)\right)$ of the coupled system

$$
\begin{gathered}
\bar{u}_{n}=\bar{u}_{n-1}+k f^{(1)}\left(\bar{u}_{n}, \underline{v}_{n}, \bar{u}_{n-s_{1}}, \underline{v}_{n-s_{2}}\right), \\
\bar{v}_{n}=\bar{v}_{n-1}+k f^{(2)}\left(\bar{u}_{n}, \bar{v}_{n}, \bar{u}_{n-s_{1}}, \bar{v}_{n-s_{2}}\right), \\
\underline{u}_{n}=\underline{u}_{n-1}+k f^{(1)}\left(\underline{u}_{n}, \bar{v}_{n}, \underline{u}_{n-s_{1}}, \bar{v}_{n-s_{2}}\right), \\
\underline{v}_{n}=\underline{v}_{n-1}+k f^{(2)}\left(\underline{u}_{n}, \underline{v}_{n}, \underline{u}_{n-s_{1}}, \underline{v}_{n-s_{2}}\right) \quad(n=1,2, \ldots), \\
\bar{u}_{n}=M^{(1)}, \quad \underline{u}_{n}=\delta^{(1)} \quad\left(n \in I_{1}\right), \quad \bar{v}_{n}=M^{(2)}, \quad \underline{v}_{n}=\delta^{(2)} \quad\left(n \in I_{2}\right) .
\end{gathered}
$$

The existence and uniqueness of a solution to (3.28) can be treated by the same argument as that for (1.1). The following theorem gives an analogous result as that in Theorem 3.3. 
Theorem 3.5. Let hypotheses $\left(H_{1}\right),\left(H_{2}\right)(c)$ and condition (3.20) be satisfied. Let also $\left(\left(\bar{u}_{n}\right.\right.$, $\left.\left.\bar{v}_{n}\right),\left(\underline{u}_{n}, \underline{v}_{n}\right)\right)$ be the solution of $(3.28)$ and $\left(u_{n}, v_{n}\right)$ the solution of $(1.1)$ with arbitrary $\left(\phi_{n}, \psi_{n}\right)$ in $\mathscr{S}$. Then

$$
\left(\underline{u}_{n}, \underline{v}_{n}\right) \leq\left(u_{n}, v_{n}\right) \leq\left(\bar{u}_{n}, \bar{v}_{n}\right) \quad(n=1,2, \ldots) .
$$

Proof. Let $\left(\bar{w}_{n}, \bar{z}_{n}\right)=\left(\bar{u}_{n}-u_{n}, \bar{v}_{n}-v_{n}\right),\left(\underline{w}_{n}, \underline{z}_{n}\right)=\left(u_{n}-\underline{u}_{n}, v_{n}-\underline{v}_{n}\right)$. By (1.1), (3.28), and the mean value theorem,

$$
\begin{aligned}
\bar{w}_{n} & =\bar{w}_{n-1}+k\left[f^{(1)}\left(\bar{u}_{n}, \underline{v}_{n}, \bar{u}_{n-s_{1}}, \underline{v}_{n-s_{2}}\right)-f^{(1)}\left(u_{n}, v_{n}, u_{n-s_{1}}, v_{n-s_{2}}\right)\right] \\
& =\bar{w}_{n-1}+\alpha_{n}^{(1)} \bar{w}_{n}-a_{n}^{(1)} \underline{z}_{n}+b_{n}^{(1)} \bar{w}_{n-s_{1}}-c_{n}^{(1)} \underline{z}_{n-s_{2}}, \\
\bar{z}_{n} & =\bar{z}_{n-1}+k\left[f^{(2)}\left(\bar{u}_{n}, \bar{v}_{n}, \bar{u}_{n-s_{1}}, \bar{v}_{n-s_{2}}\right)-f^{(2)}\left(u_{n}, v_{n}, u_{n-s_{1}}, v_{n-s_{2}}\right)\right] \\
& =\bar{z}_{n-1}+a_{n}^{(2)} \bar{w}_{n}+\alpha_{n}^{(2)} \bar{z}_{n}+b_{n}^{(2)} \bar{w}_{n-s_{1}}+c_{n}^{(2)} \bar{z}_{n-s_{2}}, \\
\underline{w}_{n} & =\underline{w}_{n-1}+k\left[f^{(1)}\left(u_{n}, v_{n}, u_{n-s_{1}}, v_{n-s_{2}}\right)-f^{(1)}\left(\underline{u}_{n}, \bar{v}_{n}, \underline{u}_{n-s_{1}}, \bar{v}_{n-s_{2}}\right)\right] \\
& =\underline{w}_{n-1}+\hat{\alpha}_{n}^{(1)} \underline{w}_{n}-\hat{a}_{n}^{(1)} \bar{z}_{n}+\hat{b}_{n}^{(1)} \underline{w}_{n-s_{1}}-\hat{c}_{n}^{(1)} \bar{z}_{n-s_{2}}, \\
\underline{z}_{n} & =\underline{z}_{n-1}+k\left[f^{(2)}\left(u_{n}, v_{n}, u_{n-s_{1}}, v_{n-s_{2}}\right)-f^{(2)}\left(\underline{u}_{n}, \underline{v}_{n}, \underline{u}_{n-s_{1}}, \underline{v}_{n-s_{2}}\right)\right] \\
& =\underline{z}_{n-1}+\hat{a}_{n}^{(2)} \underline{w}_{n}+\hat{\alpha}_{n}^{(2)} \underline{z}_{n}+\hat{b}_{n}^{(2)} \underline{w}_{n-s_{1}}+\hat{c}_{n}^{(2)} \underline{z}_{n-s_{2}} \quad(n=1,2, \ldots), \\
\bar{w}_{n} & =M^{(1)}-\phi_{n} \geq 0, \quad \underline{w}_{n}=\phi_{n}-\delta^{(1)} \geq 0 \quad\left(n \in I_{1}\right), \\
\bar{z}_{n} & =M^{(2)}-\psi_{n} \geq 0, \quad \underline{z}_{n}=\psi_{n}-\delta^{(2)} \geq 0 \quad\left(n \in I_{2}\right),
\end{aligned}
$$

where $\left(\alpha_{n}^{(l)}, a_{n}^{(l)}, b_{n}^{(l)}, c_{n}^{(l)}\right)$ and $\left(\hat{\alpha}_{n}^{(l)}, \hat{a}_{n}^{(l)}, \hat{b}_{n}^{(l)}, \hat{c}_{n}^{(l)}\right)$ are given in the form of (3.23) with possibly some different intermediate values in $\mathscr{Y}$. It is clear that the above relation can be expressed in the form (3.13) with

$$
\gamma_{n}^{(l)}=1-\alpha_{n}^{(l)}, \quad \hat{\gamma}_{n}^{(l)}=1-\hat{\alpha}_{n}^{(l)} \quad(l=1,2),
$$

and with $a_{n}^{(1)}, c_{n}^{(1)}, \hat{a}_{n}^{(1)}$, and $\hat{c}_{n}^{(1)}$ replaced, respectively, by $\left(-a_{n}^{(1)}\right),\left(-c_{n}^{(1)}\right),\left(-\hat{a}_{n}^{(1)}\right)$, and $\left(-\hat{c}_{n}^{(1)}\right)$ which are nonnegative in view of the mixed quasimonotone property (3.3). Since by hypothesis $\left(H_{2}\right)(\mathrm{c})$, all other coefficients $\left(a_{n}^{(l)}, b_{n}^{(l)}, c_{n}^{(l)}\right)$ and $\left(\hat{a}_{n}^{(l)}, \hat{b}_{n}^{(l)}, \hat{c}_{n}^{(l)}\right)$ are nonnegative, and by conditions (3.19), (3.20), and (3.23),

$$
\begin{array}{ll}
\gamma_{n}^{(1)} \geq 1-k \sigma_{1}^{(1)}>0, & \gamma_{n}^{(2)} \geq 1-k \sigma_{2}^{(2)}>0, \\
\hat{\gamma}_{n}^{(1)} \geq 1-k \sigma_{1}^{(1)}>0, & \hat{\gamma}_{n}^{(2)} \geq 1-k \sigma_{2}^{(2)}>0
\end{array}
$$

we conclude from Lemma 3.2 that $\left(\left(\bar{w}, \bar{z}_{n}\right),\left(\underline{w}_{n}, \underline{z}_{n}\right)\right) \geq((0,0),(0,0))$ if condition $(3.14)$ holds. Since

$$
0 \leq\left(-a_{n}^{(1)}\right) \leq k \sigma_{2}^{(1)}, \quad a_{n}^{(2)} \leq k \sigma_{1}^{(2)}, \quad 0 \leq\left(-\hat{a}_{n}^{(1)}\right) \leq k \sigma_{2}^{(1)}, \quad \hat{a}_{n}^{(2)} \leq k \sigma_{1}^{(2)},
$$


we see from (3.31) and (3.32) that condition (3.14) holds if

$$
\left[\left(k \sigma_{2}^{(1)}\right)\left(k \sigma_{1}^{(2)}\right)\right]^{2}<\left[\left(1-k \sigma_{1}^{(1)}\right)\left(1-k \sigma_{2}^{(2)}\right)\right]^{2} .
$$

But the above inequality is equivalent to (3.20). This proves relation (3.29) and thus the theorem.

\section{Asymptotic behavior}

It is seen from the comparison theorems in the previous section that the asymptotic behavior of the solution $\left(u_{n}, v_{n}\right)$ for arbitrary initial functions in $\mathscr{Y}$ can be determined from the behavior of the solutions $\left(\bar{u}_{n}, \bar{v}_{n}\right)$ and $\left(\underline{u}_{n}, \underline{v}_{n}\right)$ provided that these solutions converge to a common limit as $n \rightarrow \infty$. In this section, we show the monotone convergence of $\left(\bar{u}_{n}, \bar{v}_{n}\right)$ and $\left(\underline{u}_{n}, \underline{v}_{n}\right)$ to equilibrium solutions (or quasiequilibrium solutions) of (1.1) for each of the three types of quasimonotone functions $\left(f^{(1)}, f^{(2)}\right)$. Here by an equilibrium solution (or simply equilibrium), we mean a constant $(u, v) \in \mathscr{Y}$ such that

$$
f^{(1)}(u, v, u, v)=0, \quad f^{(2)}(u, v, u, v)=0 .
$$

Our first result is for quasimonotone nondecreasing functions.

Theorem 4.1. Let the conditions in Theorem 3.3 be satisfied. Then the solution $\left(\bar{u}_{n}, \bar{v}_{n}\right)$ converges monotonically to a maximal equilibrium $(\bar{u}, \bar{v})$, the solution $\left(\underline{u}_{n}, \underline{v}_{n}\right)$ converges monotonically to a minimal equilibrium $(\underline{u}, \underline{v})$, and

$$
\left(\delta^{(1)}, \delta^{(2)}\right) \leq(\underline{u}, \underline{v}) \leq(\bar{u}, \bar{v}) \leq\left(M^{(1)}, M^{(2)}\right) .
$$

If, in addition, $(\bar{u}, \bar{v})=(\underline{u}, \underline{v})\left(\equiv\left(u^{*}, v^{*}\right)\right)$, then $\left(u^{*}, v^{*}\right)$ is the unique equilibrium in $\mathscr{Y}$, and for arbitrary $\left(\phi_{n}, \psi_{n}\right)$ in $\mathscr{S}$ the corresponding solution $\left(u_{n}, v_{n}\right)$ converges to $\left(u^{*}, v^{*}\right)$ as $n \rightarrow \infty$.

Proof. Consider the solution $\left(\underline{u}_{n}, \underline{v}_{n}\right)$, and let $\left(w_{n}, z_{n}\right)=\left(\underline{u}_{n+1}-\underline{u}_{n}, \underline{v}_{n+1}-\underline{v}_{n}\right)$. By (1.1) and the mean value theorem,

$$
\begin{aligned}
w_{n} & =w_{n-1}+k\left[f^{(1)}\left(\underline{u}_{n+1}, \underline{v}_{n+1}, \underline{u}_{n+1-s_{1}}, \underline{v}_{n+1-s_{2}}\right)-f^{(1)}\left(\underline{u}_{n}, \underline{v}_{n}, \underline{u}_{n-s_{1}}, \underline{v}_{n-s_{2}}\right)\right] \\
& =w_{n-1}+\alpha_{n}^{(1)} w_{n}+a_{n}^{(1)} z_{n}+b_{n}^{(1)} w_{n-s_{1}}+c_{n}^{(1)} z_{n-s_{2}}, \\
z_{n} & =z_{n-1}+k\left[f^{(2)}\left(\underline{u}_{n+1}, \underline{v}_{n+1}, \underline{u}_{n+1-s_{1}}, \underline{v}_{n+1-s_{2}}\right)-f^{(2)}\left(\underline{u}_{n}, \underline{v}_{n}, \underline{u}_{n-s_{1}}, \underline{v}_{n-s_{2}}\right)\right] \\
& =z_{n-1}+a_{n}^{(2)} w_{n}+\alpha_{n}^{(2)} z_{n}+b_{n}^{(2)} w_{n-s_{1}}+c_{n}^{(2)} z_{n-s_{2}} \quad(n=1,2, \ldots), \\
w_{n} & =\underline{u}_{n+1}-\delta^{(1)} \geq 0 \quad\left(n \in I_{1}\right), \quad z_{n}=\underline{v}_{n+1}-\delta^{(2)} \geq 0 \quad\left(n \in I_{2}\right),
\end{aligned}
$$

where $\alpha_{n}^{(l)}, a_{n}^{(l)}, b_{n}^{(l)}$, and $c_{n}^{(l)}$ are given by (3.23) with possibly some different intermediate values $\xi_{n}, \boldsymbol{\xi}_{n}^{\prime}$. Since $\left(\underline{u}_{n}, \underline{v}_{n}\right) \in \mathscr{S}$ for all $n$, the values of $\boldsymbol{\xi}_{n}$ and $\boldsymbol{\xi}_{n}^{\prime}$ remain in $\mathscr{T}$, and therefore the coefficients $a_{n}^{(l)}, b_{n}^{(l)}$, and $c_{n}^{(l)}$ are nonnegative. It follows from the proof 
of Theorem 3.3 that $\left(w_{n}, z_{n}\right) \geq(0,0)$ for $n=1,2, \ldots$. This proves the relation $\left(\underline{u}_{n}, \underline{v}_{n}\right) \leq$ $\left(\underline{u}_{n+1}, \underline{v}_{n+1}\right)$. A similar argument gives $\left(\bar{u}_{n+1}, \bar{v}_{n+1}\right) \leq\left(\bar{u}_{n}, \bar{v}_{n}\right)$ and $\left(\underline{u}_{n}, \underline{v}_{n}\right) \leq\left(\bar{u}_{n}, \bar{v}_{n}\right)$ for every $n=1,2, \ldots$.

This leads to the relation

$$
\underline{u}_{n} \leq \underline{u}_{n+1} \leq \bar{u}_{n+1} \leq \bar{u}_{n}, \quad \underline{v}_{n} \leq \underline{v}_{n+1} \leq \bar{v}_{n+1} \leq \bar{v}_{n}, \quad n=1,2, \ldots
$$

The above monotone property ensures that the limits

$$
\lim _{n \rightarrow \infty}\left(\bar{u}_{n}, \bar{v}_{n}\right)=(\bar{u}, \bar{v}), \quad \lim _{n \rightarrow \infty}\left(\underline{u}_{n}, \underline{v}_{n}\right)=(\underline{u}, \underline{v})
$$

exist and satisfy relation (4.2). Letting $n \rightarrow \infty$ in (1.1) shows that $(\bar{u}, \bar{v})$ and $(\underline{u}, \underline{v})$ are solutions of (4.1). Now if $(u, v)$ is another solution of $(4.1)$ in $\mathscr{Y}$, then by considering $(u, v)$ as a solution of $(1.1)$ with $\left(\phi_{n}, \psi_{n}\right)=(u, v)$, Theorem 3.3 ensures that $\left(\underline{u}_{n}, \underline{v}_{n}\right) \leq$ $(u, v) \leq\left(\bar{u}_{n}, \bar{v}_{n}\right)$ for every $n$. Letting $n \rightarrow \infty$ yields $(\underline{u}, \underline{v}) \leq(u, v) \leq(\bar{u}, \bar{v})$. This proves the maximal and minimal property of $(\bar{u}, \bar{v})$ and $(\underline{u}, \underline{v})$, respectively. It is clear that if $(\bar{u}, \bar{v})=$ $(\underline{u}, \underline{v})\left(\equiv\left(u^{*}, v^{*}\right)\right)$, then the above maximal and minimal property implies that $\left(u^{*}, v^{*}\right)$ is the unique equilibrium solution in $\mathscr{Y}$. Moreover, by Theorem 3.3, $\left(u_{n}, v_{n}\right) \rightarrow\left(u^{*}, v^{*}\right)$ as $n \rightarrow \infty$. The proof of the theorem is completed.

If $f^{(1)} \equiv f^{(1)}(u)$ is independent of $\left(v, u_{s}, v_{s}\right)$ and there exist constants $M \geq \delta>0$ such that

$$
f^{(1)}(M) \leq 0 \leq f^{(1)}(\delta)
$$

then all the conditions in $\left(H_{1}\right)$ and $\left(H_{2}\right)($ a) for the scalar problem

$$
u_{n}=u_{n-1}+k f^{(1)}\left(u_{n}\right), \quad u_{0}=\phi \quad(n=1,2, \ldots)
$$

are satisfied. In this situation, Theorem 4.1 ensures that the solutions $\bar{u}_{n}, \underline{u}_{n}$ of (4.7) with $\phi=M$ and $\phi=\delta$, respectively, converge to some constants $\bar{u}$ and $\underline{u}$ as $n \rightarrow \infty$. Moreover, $\bar{u}$ and $\underline{u}$ satisfy the equation $f^{(1)}(\bar{u})=f^{(1)}(\underline{u})=0$ and the relation $\delta \leq \underline{u} \leq \bar{u} \leq M$. If $\bar{u}=\underline{u}\left(\equiv u^{*}\right)$, then for any $\phi \in[\delta, M]$, the corresponding solution $u_{n}$ of (4.7) converges to $u^{*}$ as $n \rightarrow \infty$. In particular, if $f^{(1)}(u)=\alpha u(1-\beta u)$ for some positive constants $\alpha$, $\beta$, then condition (4.6) is fulfilled by any constants $M, \delta$ satisfying $0<\delta \leq \beta^{-1} \leq M$, and the limits $\bar{u}, \underline{u}$ are both equal to $\beta^{-1}$. Since $\delta$ can be chosen arbitrarily small and $M$ arbitrarily large, we have the following result which will be needed in later applications.

Corollary 4.2. If $f^{(1)} \equiv f^{(1)}(u)$ is a $C^{1}$-function such that $k \sigma_{1}^{(1)}<1$ and condition (4.6) holds for some constants $M \geq \delta>0$, then the solutions $\bar{u}_{n}, \underline{u}_{n}$ of (4.7) with $\phi=M$ and $\phi=\delta$, respectively, converge to some equilibrium solutions $\bar{u}$, $\underline{u}$ such that $f^{(1)}(\bar{u})=f^{(1)}(\underline{u})=0$. In particular, if $f^{(1)}(u)=\alpha u(1-\beta u)$ for some positive constants $\alpha$, then for any $\phi>0$, the corresponding solution $u_{n}$ of (4.7) converges to $\beta^{-1}$ as $n \rightarrow \infty$.

For quasimonotone nonincreasing functions, we have the following analogous result.

Theorem 4.3. Let the conditions in Theorem 3.4 be satisfied. Then the solution $\left(\bar{u}_{n}, \underline{v}_{n}\right)$ converges monotonically to an equilibrium $(\bar{u}, \underline{v})$, the solution $\left(\underline{u}_{n}, \bar{v}_{n}\right)$ converges monotonically 
to an equilibrium $(\underline{u}, \bar{v})$, and

$$
\delta^{(1)} \leq \underline{u} \leq \bar{u} \leq M^{(1)}, \quad \delta^{(2)} \leq \underline{v} \leq \bar{v} \leq M^{(2)} .
$$

If, in addition, $(\bar{u}, \underline{v})=(\underline{u}, \bar{v})\left(\equiv\left(u^{*}, v^{*}\right)\right)$, then $\left(u^{*}, v^{*}\right)$ is the unique equilibrium in $\mathscr{S}$ and for any $\left(\phi_{n}, \psi_{n}\right) \in \mathscr{S}$, the corresponding solution $\left(u_{n}, v_{n}\right)$ converges to $\left(u^{*}, v^{*}\right)$ as $n \rightarrow \infty$.

Proof. Consider the solution $\left(\bar{u}_{n}, \underline{v}_{n}\right)$, and let $\left(w_{n}, z_{n}\right)=\left(\bar{u}_{n}-\bar{u}_{n+1}, \underline{v}_{n+1}-\underline{v}_{n}\right)$. Then,

$$
\begin{aligned}
w_{n} & =w_{n-1}+k\left[f^{(1)}\left(\bar{u}_{n}, \underline{v}_{n}, \bar{u}_{n-s_{1}}, \underline{v}_{n-s_{2}}\right)-f^{(1)}\left(\bar{u}_{n+1}, \underline{v}_{n+1}, \bar{u}_{n+1-s_{1}}, \underline{v}_{n+1-s_{2}}\right)\right] \\
& =w_{n-1}+\alpha_{n}^{(1)} w_{n}-a_{n}^{(1)} z_{n}+b_{n}^{(1)} w_{n-s_{1}}-c_{n}^{(1)} z_{n-s_{2}}, \\
z_{n} & =z_{n-1}+k\left[f^{(2)}\left(\bar{u}_{n+1}, \underline{v}_{n+1}, \bar{u}_{n+1-s_{1}}, \underline{v}_{n+1-s_{2}}\right)-f^{(2)}\left(\bar{u}_{n}, \underline{v}_{n}, \bar{u}_{n-s_{1}}, \underline{v}_{n-s_{2}}\right)\right] \\
& =z_{n-1}-a_{n}^{(2)} w_{n}+\alpha_{n}^{(2)} z_{n}-b_{n}^{(2)} w_{n-s_{1}}+c_{n}^{(2)} z_{n-s_{2}} \quad(n=1,2, \ldots), \\
w_{n} & =M^{(1)}-\bar{u}_{n+1} \geq 0 \quad\left(n \in I_{1}\right), \quad z_{n}=\underline{v}_{n+1}-\delta^{(2)} \geq 0 \quad\left(n \in I_{2}\right),
\end{aligned}
$$

where $\alpha_{n}^{(l)}, a_{n}^{(l)}, b_{n}^{(l)}$, and $c_{n}^{(l)}$ are given by (3.23) with possibly some different $\xi$, $\xi^{\prime}$ in $\mathscr{S} \times \mathscr{Y}$. Since relation (4.9) is the same as that in (3.27), the reasoning in the proof of Theorem 3.4 shows that $\bar{u}_{n} \geq \bar{u}_{n+1}$ and $\underline{v}_{n+1} \geq \underline{v}_{n}$. A similar argument gives $\underline{u}_{n+1} \geq \underline{u}_{n}$ and $\bar{v}_{n} \geq \bar{v}_{n+1}$. This shows that $\left(\bar{u}_{n}, \underline{v}_{n}\right)$ and $\left(\underline{u}_{n}, \bar{v}_{n}\right)$ possess the monotone property (4.4). Hence, the limits

$$
\lim _{n \rightarrow \infty}\left(\bar{u}_{n}, \underline{v}_{n}\right)=(\bar{u}, \underline{v}), \quad \lim _{n \rightarrow \infty}\left(\underline{u}_{n}, \bar{v}_{n}\right)=(\underline{u}, \bar{v})
$$

exist and are equilibrium solutions in $\mathscr{Y}$. If $(\bar{u}, \underline{v})=(\underline{u}, \bar{v})\left(\equiv\left(u^{*}, v^{*}\right)\right)$, then the uniqueness of the equilibrium $\left(u^{*}, v^{*}\right)$ and the convergence of $\left(u_{n}, v_{n}\right)$ to $\left(u^{*}, v^{*}\right)$ follow from $(3.26)$ by letting $n \rightarrow \infty$. This proves the theorem.

For mixed quasimonotone functions, we say that the constants $(\bar{u}, \bar{v}),(\underline{u}, \underline{v})$ are a pair of quasiequilibrium solutions of $(1.1)$ if $(\bar{u}, \bar{v})$ and $(\underline{u}, \underline{v})$ are in $\mathscr{Y}$ and if

$$
\begin{array}{ll}
f^{(1)}(\bar{u}, \underline{v}, \bar{u}, \underline{v})=0, & f^{(1)}=(\underline{u}, \bar{v}, \underline{u}, \bar{v})=0, \\
f^{(2)}(\bar{u}, \bar{v}, \bar{u}, \bar{v})=0, & f^{(2)}=(\underline{u}, \underline{v}, \underline{u}, \underline{v})=0 .
\end{array}
$$

It is obvious that quasiequilibrium solutions are not necessarily true equilibrium solutions unless $\bar{u}=\underline{u}$ or $\bar{v}=\underline{v}$. In the following theorem, we show the convergence of the solution of (3.28) to quasiequilibrium solutions.

Theorem 4.4. Let the conditions in Theorem 3.5 be satisfied. Then the solution $\left(\left(\bar{u}_{n}, \bar{v}_{n}\right)\right.$, $\left.\left(\underline{u}_{n}, \underline{v}_{n}\right)\right)$ of (3.28) converges monotonically to a pair of quasiequilibrium solutions $((\bar{u}, \bar{v})$, $(\underline{u}, \underline{v}))$ that satisfy $(4.11)$. If $(\bar{u}, \bar{v})=(\underline{u}, \underline{v})\left(\equiv\left(u^{*}, v^{*}\right)\right)$, then $\left(u^{*}, v^{*}\right)$ is the unique equilibrium in $\mathscr{S}$ and for any $\left(\phi_{n}, \psi_{n}\right) \in \mathscr{S}$, the corresponding solution $\left(u_{n}, v_{n}\right)$ of $(1.1)$ converges to $\left(u^{*}, v^{*}\right)$ as $n \rightarrow \infty$. 
Proof. Let $\left(\bar{w}_{n}, \bar{z}_{n}\right)=\left(\bar{u}_{n}-\bar{u}_{n+1}, \bar{v}_{n}-\bar{v}_{n+1}\right),\left(\underline{w}_{n}, \underline{z}_{n}\right)=\left(\underline{u}_{n+1}-\underline{u}_{n}, \underline{v}_{n+1}-\underline{v}_{n}\right)$. Вy (3.28) and the mean-value theorem,

$$
\begin{aligned}
\bar{w}_{n}= & \bar{w}_{n-1}+k\left[f^{(1)}\left(\bar{u}_{n}, \underline{v}_{n}, \bar{u}_{n-s_{1}}, \underline{v}_{n-s_{2}}\right)-f^{(1)}\left(\bar{u}_{n+1}, \underline{v}_{n+1}, \bar{u}_{n+1-s_{1}}, \underline{v}_{n+1-s_{2}}\right)\right] \\
= & \bar{w}_{n-1}+\alpha_{n}^{(1)} \bar{w}_{n}-a_{n}^{(1)} \underline{z}_{n}+b_{n}^{(1)} \bar{w}_{n-s_{1}}-c_{n}^{(1)} \underline{z}_{n-s_{2}}, \\
\bar{z}_{n}= & \bar{z}_{n-1}+k\left[f^{(2)}\left(\bar{u}_{n}, \bar{v}_{n}, \bar{u}_{n-s_{1}}, \bar{v}_{n-s_{2}}\right)-f^{(2)}\left(\bar{u}_{n+1}, \bar{v}_{n+1}, \bar{u}_{n+1-s_{1}}, \bar{v}_{n+1-s_{2}}\right)\right] \\
= & \bar{z}_{n-1}+a_{n}^{(2)} \bar{w}_{n}+\alpha_{n}^{(2)} \bar{z}_{n}+b_{n}^{(2)} \bar{w}_{n-s_{1}}+c_{n}^{(2)} \bar{z}_{n-s_{2}}, \\
\underline{w}_{n}= & \underline{w}_{n-1}+k\left[f^{(1)}\left(\underline{u}_{n+1}, \bar{v}_{n+1}, \underline{u}_{n+1-s_{1}}, \bar{v}_{n+1-s_{2}}\right)-f^{(1)}\left(\underline{u}_{n}, \bar{v}_{n}, \underline{u}_{n-s_{1}}, \bar{v}_{n-s_{2}}\right)\right] \\
= & \underline{w}_{n-1}+\hat{\alpha}_{n}^{(1)} \underline{w}_{n}-\hat{a}_{n}^{(1)} \bar{z}_{n}+\hat{b}_{n}^{(1)} \underline{w}_{n-s_{1}}-\hat{c}_{n}^{(1)} \bar{z}_{n-s_{2}}, \quad \\
\underline{z}_{n}= & \underline{z}_{n-1}+k\left[f^{(2)}\left(\underline{u}_{n+1}, \underline{v}_{n+1}, \underline{u}_{n+1-s_{1}}, \underline{v}_{n+1-s_{2}}\right)-f^{(2)}\left(\underline{u}_{n}, \underline{v}_{n}, \underline{u}_{n-s_{1}}, \underline{v}_{n-s_{2}}\right)\right] \\
= & \underline{z}_{n-1}+\hat{a}_{n}^{(2)} \underline{w}_{n}+\hat{\alpha}_{n}^{(2)} \underline{z}_{n}+\hat{b}_{n}^{(2)} \underline{w}_{n-s_{1}}+\hat{c}_{n}^{(2)} \underline{z}_{n-s_{2}} \quad(n=1,2, \ldots), \\
& \bar{w}_{n} \geq 0, \quad \underline{w}_{n} \geq 0 \quad\left(n \in I_{1}\right), \quad \bar{z}_{n} \geq 0, \quad \underline{z}_{n} \geq 0 \quad\left(n \in I_{2}\right) .
\end{aligned}
$$

Since the above relation is in the same form as that in (3.30), we conclude from the proof of Theorem 3.5 that $\left(\left(\bar{w}_{n}, \bar{z}_{n}\right),\left(\underline{w}_{n}, \underline{z}_{n}\right)\right) \geq((0,0),(0,0))$. This proves the monotone property (4.4) for $\left(\left(\bar{u}_{n}, \bar{v}_{n}\right),\left(\underline{u}_{n}, \underline{v}_{n}\right)\right)$. It follows from this property that the limits $(\bar{u}, \bar{v}),(\underline{u}, \underline{v})$ in (4.5) exist and satisfy (4.2). Letting $n \rightarrow \infty$ in (3.28) shows that these limits satisfy the equations in (4.11). If $(\bar{u}, \bar{v})=(\underline{u}, \underline{v})\left(\equiv\left(u^{*}, v^{*}\right)\right)$, then $\left(u^{*}, v^{*}\right)$ is a solution of $(4.1)$, and both $\left(\bar{u}_{n}, \bar{v}_{n}\right)$ and $\left(\underline{u}_{n}, \underline{v}_{n}\right)$ converge to $\left(u^{*}, v^{*}\right)$ as $n \rightarrow \infty$. The uniqueness of $\left(u^{*}, v^{*}\right)$ in $\mathscr{Y}$ and the convergence of $\left(u_{n}, v_{n}\right)$ to $\left(u^{*}, v^{*}\right)$ follow from Theorem 3.5.

The previous theorems imply that for each type of quasimonotone functions if $(\bar{u}, \bar{v})=$ $(\underline{u}, \underline{v}) \equiv\left(u^{*}, v^{*}\right)$, then $\left(u^{*}, v^{*}\right)$ is a global attractor relative to the set $\mathscr{S}$. In the following theorem, we give a sufficient condition for the global attraction of $\left(u^{*}, v^{*}\right)$ relative to the whole space $\mathbb{R}^{2}$ (or the positive cone $\mathbb{R}_{+}^{2}$ ) for any of the three types of quasimonotone functions.

THeORem 4.5. Let the conditions in Theorems 3.3, 3.4, and 3.5 be satisfied for the respective quasimonotone functions, and let $\left(u_{n}, v_{n}\right)$ be the solution of (1.1) for an arbitrary initial function $\left(\phi_{n}, \psi_{n}\right)$, not necessarily in $\mathscr{Y}$. Assume that $\left(\bar{u}_{n}, \bar{v}_{n}\right)=\left(\underline{u}_{n}, \underline{v}_{n}\right)\left(\equiv\left(u^{*}, v^{*}\right)\right)$. If there exists $n_{0}>0$ such that

$$
\left(\delta^{(1)}, \delta^{(2)}\right) \leq\left(u_{n}, v_{n}\right) \leq\left(M^{(1)}, M^{(2)}\right) \quad \text { for } n_{0}-\bar{s} \leq n \leq n_{0}
$$

where $\bar{s}=\max \left\{s_{1}, s_{2}\right\}$, then

$$
\lim \left(u_{n}, v_{n}\right)=\left(u^{*}, v^{*}\right) \text { as } n \longrightarrow \infty
$$


Proof. Let $\left(w_{n}, z_{n}\right)=\left(u_{n+n_{0}}, v_{n+n_{0}}\right)$. Then by $(1.1)$,

$$
\begin{aligned}
w_{n} & =u_{n+n_{0}-1}+k f^{(1)}\left(u_{n+n_{0}}, v_{n+n_{0}}, u_{n+n_{0}-s_{1}}, v_{n+n_{0}-s_{2}}\right) \\
& =w_{n-1}+k f^{(1)}\left(w_{n}, z_{n}, w_{n-s_{1}}, z_{n-s_{2}}\right), \\
z_{n} & =v_{n+n_{0}-1}+k f^{(2)}\left(u_{n+n_{0}}, v_{n+n_{0}}, u_{n+n_{0}-s_{1}}, v_{n+n_{0}-s_{2}}\right) \\
& =z_{n-1}+k f^{(2)}\left(w_{n}, z_{n}, w_{n-s_{1}}, z_{n-s_{2}}\right) \quad(n=1,2, \ldots) .
\end{aligned}
$$

Since by (4.13), $\left(w_{n}, z_{n}\right) \in \mathscr{S}$ for $-\bar{s} \leq n \leq 0$, we conclude by applying Theorems 4.1, 4.3 , and 4.4 to $\left(w_{n}, z_{n}\right)$ for the corresponding quasimonotone function that $\left(w_{n}, z_{n}\right) \rightarrow$ $\left(u^{*}, v^{*}\right)$ as $n \rightarrow \infty$. This leads to relation (4.14).

When $f^{(l)}\left(u_{n}, v_{n}, u_{n-s_{1}}, v_{n-s_{2}}\right)$ is independent of $u_{n-s_{1}}$ or $v_{n-s_{2}}$ (or both) for $l=1$ or $l=2$ (or both), all the requirements in $\left(H_{1}\right),\left(H_{2}\right)$ with respect to the variables $u_{n-s_{1}}$ and $v_{n-s_{2}}$ are fulfilled. In particular, if $f^{(l)} \equiv f^{(l)}\left(u_{n}, v_{n}\right)$ is independent of $\left(u_{n-s_{1}}, v_{n-s_{2}}\right)$ for both $l=1$ and $l=2$, then system (1.1) is reduced to the standard initial-value problem

$$
\begin{gathered}
u_{n}=u_{n-1}+k f^{(1)}\left(u_{n}, v_{n}\right), \quad u_{0}=\phi, \\
v_{n}=v_{n-1}+k f^{(2)}\left(u_{n}, v_{n}\right), \quad v_{0}=\psi(n=1,2, \ldots) .
\end{gathered}
$$

In this situation, the definition of quasimonotone functions is reduced to the standard one (i.e., with $\partial f^{(l)} / \partial u_{s}=\partial f^{(l)} / \partial v_{s}=0$ for $l=1,2$ in $\left.\left(H_{2}\right)\right)$, and condition (2.2) in $\left(H_{1}\right)$ becomes

$$
\begin{array}{ll}
f^{(1)}\left(M^{(1)}, v\right) \leq 0 \leq f^{(1)}\left(\delta^{(1)}, v\right) & \text { for } \delta^{(2)} \leq v \leq M^{(2)}, \\
f^{(2)}\left(u, M^{(2)}\right) \leq 0 \leq f^{(2)}\left(u, \delta^{(1)}\right) & \text { for } \delta^{(1)} \leq u \leq M^{(1)} .
\end{array}
$$

In this special case, all the conclusions of Theorem 4.1 to Theorem 4.5 are directly applicable to (4.16). Because of its usefulness in applications, we state the above results in the following theorem.

Theorem 4.6. Let $\left(f^{(1)}, f^{(2)}\right) \equiv\left(f^{(1)}\left(u_{n}, v_{n}\right), f^{(2)}\left(u_{n}, v_{n}\right)\right)$ satisfy hypotheses $\left(H_{1}\right),\left(H_{2}\right)$ with condition (2.2) replaced by (4.17), and let $k$ satisfy condition $(3.20)$. If $(\bar{u}, \bar{v})=(\underline{u}, \underline{v})$ $\left(\equiv\left(u^{*}, v^{*}\right)\right)$, then $\left(u^{*}, v^{*}\right)$ is the unique positive equilibrium in $\mathscr{Y}$, and for any $(\phi, \psi) \in \mathscr{Y}$, the corresponding solution $\left(u_{n}, v_{n}\right)$ of $(4.16)$ converges to $\left(u^{*}, v^{*}\right)$ as $n \rightarrow \infty$. Moreover, this convergence property holds true for the solution $\left(u_{n}, v_{n}\right)$ corresponding to an arbitrary $(\phi, \psi)$ if condition (4.13) holds for some $n_{0}>0$.

\section{Applications to Lotka-Volterra systems}

In this section, we give some applications of the global stability results in the previous section to the three Lotka-Volterra model problems (1.4), (1.5), and (1.6). It is clear that in these models the reaction function $\left(f^{(1)}, f^{(2)}\right)$ is quasimonotone nondecreasing, quasimonotone nonincreasing, and mixed quasimonotone, respectively, in $\mathscr{S} \times \mathscr{S}$ for every $M^{(l)} \geq \delta^{(l)} \geq 0, l=1,2$. To apply the theorems in Section 4 , we need

(i) to find some positive constants $\left(M^{(1)}, M^{(2)}\right),\left(\delta^{(1)}, \delta^{(2)}\right)$ satisfying condition (2.2), or the corresponding conditions (3.4), (3.5), and (3.6); 
(ii) to show that the limits $(\bar{u}, \bar{v}),(\underline{u}, \underline{v})$ in $(4.5)$ or in (4.10) coincide;

(iii) to verify that the solution $\left(u_{n}, v_{n}\right)$ corresponding to an arbitrary nonnegative $\left(\phi_{n}, \psi_{n}\right)$ with $\left(\phi_{0}, \psi_{0}\right)>(0,0)$ converges to $\left(u^{*}, v^{*}\right)$ as $n \rightarrow \infty$.

To do this for each of the three models, we set

$$
\bar{a}^{(2)}=a^{(2)}+c^{(2)}, \quad \bar{b}^{(1)}=b^{(1)}+c^{(1)}, \quad \Delta=a^{(1)} b^{(2)}-\bar{a}^{(2)} \bar{b}^{(1)},
$$

and assume that $k$ satisfies condition (3.20) with respect to the function $\left(f^{(1)}, f^{(2)}\right)$ in (1.4), (1.5), or (1.6). Our purpose is to obtain a sufficient condition on the four constants $a^{(1)}, b^{(2)}, \bar{a}^{(2)}$, and $\bar{b}^{(1)}$, so that a unique positive equilibrium solution exists and is a global attractor in the positive cone $\mathbb{R}_{+}^{2}$.

5.1. The cooperative model. (i) In the cooperative model (1.4), the reaction function $\left(f^{(1)}, f^{(2)}\right)$ is given by

$$
\begin{aligned}
& f^{(1)}\left(u_{n}, v_{n}, v_{n-s_{2}}\right)=\alpha^{(1)} u_{n}\left(1-a^{(1)} u_{n}+b^{(1)} v_{n}+c^{(1)} v_{n-s_{2}}\right), \\
& f^{(2)}\left(u_{n}, v_{n}, u_{n-s_{1}}\right)=\alpha^{(2)} v_{n}\left(1+a^{(2)} u_{n}-b^{(2)} v_{n}+c^{(2)} u_{n-s_{1}}\right) .
\end{aligned}
$$

It is obvious that the above function is quasimonotone nondecreasing in $\mathscr{Y} \times \mathscr{Y}$ and satisfies hypothesis $\left(H_{2}\right)(\mathrm{a})$. Moreover, the requirement on $\left(M^{(1)}, M^{(2)}\right),\left(\delta^{(1)}, \delta^{(2)}\right)$ in $(3.4)$ (or (2.2)) becomes

$$
\begin{aligned}
& \alpha^{(1)} M^{(1)}\left(1-a^{(1)} M^{(1)}+\bar{b}^{(1)} M^{(2)}\right) \leq 0 \leq \alpha^{(1)} \delta^{(1)}\left(1-a^{(1)} \delta^{(1)}+\bar{b}^{(1)} \delta^{(2)}\right), \\
& \alpha^{(2)} M^{(2)}\left(1+\bar{a}^{(2)} M^{(1)}-b^{(2)} M^{(2)}\right) \leq 0 \leq \alpha^{(2)} \delta^{(2)}\left(1+\bar{a}^{(2)} \delta^{(1)}-b^{(2)} \delta^{(2)}\right) .
\end{aligned}
$$

The above inequalities are satisfied by a small $\left(\delta^{(1)}, \delta^{(2)}\right)>(0,0)$ if

$$
a^{(1)} M^{(1)}-\bar{b}^{(1)} M^{(2)} \geq 1, \quad-\bar{a}^{(2)} M^{(1)}+b^{(2)} M^{(2)} \geq 1 .
$$

Assume $\Delta>0$, where $\Delta$ is given by (5.1). Then the system

$$
a^{(1)} u-\bar{b}^{(1)} v=1, \quad-\bar{a}^{(2)} u+b^{(2)} v=1
$$

has a unique positive solution given by

$$
\left(u^{*}, v^{*}\right)=\left(\frac{\bar{b}^{(1)}+b^{(2)}}{\Delta}, \frac{a^{(1)}+\bar{a}^{(2)}}{\Delta}\right) .
$$

We choose $\left(M^{(1)}, M^{(2)}\right)=\left(\rho u^{*}, \rho v^{*}\right)$ for some $\rho \geq 1$. It is obvious that $\left(M^{(1)}, M^{(2)}\right)$ satisfies (5.4) for any choice of $\rho \geq 1$. This shows that the pair $\left(M^{(1)}, M^{(2)}\right),\left(\delta^{(1)}, \delta^{(2)}\right)$ fulfills the requirement in (3.4). 
(ii) Since hypotheses $\left(H_{1}\right)$ and $\left(H_{2}\right)($ a) are satisfied, Theorem 4.1 implies that the solutions $\left(\bar{u}_{n}, \bar{v}_{n}\right),\left(\underline{u}_{n}, \underline{v}_{n}\right)$ converge to some limits $(\bar{u}, \bar{v}),(\underline{u}, \underline{v})$ that satisfy the relation

$$
\begin{array}{ll}
1-a^{(1)} \bar{u}+\bar{b}^{(1)} \bar{v}=0, & 1+\bar{a}^{(2)} \bar{u}-b^{(2)} \bar{v}=0, \\
1-a^{(1)} \underline{u}+\bar{b}^{(1)} \underline{v}=0, & 1+\bar{a}^{(2)} \underline{u}-b^{(2)} \underline{v}=0 .
\end{array}
$$

A subtraction of the above equations leads to

$$
a^{(1)}(\bar{u}-\underline{u})-\bar{b}^{(1)}(\bar{v}-\underline{v})=0, \quad-\bar{a}^{(2)}(\bar{u}-\underline{u})+b^{(2)}(\bar{v}-\underline{v})=0 .
$$

It follows from $\Delta \neq 0$ that $\bar{u}-\underline{u}=\bar{v}-\underline{v}=0$. This shows that $(\bar{u}, \bar{v})=(\underline{u}, \underline{v})=\left(u^{*}, v^{*}\right)$ and $\left(u^{*}, v^{*}\right)$ is the unique positive equilibrium given by (5.6). By Theorem 4.1, the solution $\left(u_{n}, v_{n}\right)$ corresponding to any $\left(\phi_{n}, \psi_{n}\right) \in \mathscr{Y}$ converges to $\left(u^{*}, v^{*}\right)$ as $n \rightarrow \infty$.

(iii) To show the convergence of $\left(u_{n}, v_{n}\right)$ to $\left(u^{*}, v^{*}\right)$ for an arbitrary nonnegative $\left(\phi_{n}\right.$, $\left.\psi_{n}\right)$ with $\left(\phi_{0}, \psi_{0}\right)>(0,0)$, we choose $\rho \geq 1$ such that $\left(M^{(1)}, M^{(2)}\right)=\left(\rho u^{*}, \rho v^{*}\right) \geq\left(\phi_{n}, \psi_{n}\right)$ and satisfies the left-hand side inequalities in (5.3). This implies that $\left(u_{n}, v_{n}\right) \leq\left(M^{(1)}, M^{(2)}\right)$ for all $n=1,2, \ldots$ It is easy to see that if $\left(\phi_{0}, \psi_{0}\right)>(0,0)$, then $\left(u_{n}, v_{n}\right)$ is positive for every $n$, and therefore there exists $n_{0}>0$ and a sufficiently small $\left(\delta^{(1)}, \delta^{(2)}\right)>(0,0)$ such that $\left(u_{n}, v_{n}\right) \geq\left(\delta^{(1)}, \delta^{(2)}\right)$ for $n_{0}-\bar{s} \leq n \leq n_{0}$. This ensures that $\left(u_{n}, v_{n}\right)$ satisfies condition (4.13). The convergence of $\left(u_{n}, v_{n}\right)$ to $\left(u^{*}, v^{*}\right)$ follows from Theorem 4.5. To summarize the above conclusions, we have the following result.

Theorem 5.1. Let $\Delta \equiv a^{(1)} b^{(2)}-\bar{a}^{(2)} \bar{b}^{(1)}>0$ and $k$ satisfy condition (3.20), and let $\left(u^{*}, v^{*}\right)$ be the positive equilibrium given by (5.6). Then for any nonnegative $\left(\phi_{n}, \psi_{n}\right)$ with $\left(\phi_{0}, \psi_{0}\right)>$ $(0,0)$, the corresponding solution $\left(u_{n}, v_{n}\right)$ of $(1.4)$ converges to $\left(u^{*}, v^{*}\right)$ as $n \rightarrow \infty$.

5.2. The competition model. (i) In the competition model (1.5), the reaction function $\left(f^{(1)}, f^{(2)}\right)$ is

$$
\begin{aligned}
& f^{(1)}\left(u_{n}, v_{n}, v_{n-s_{2}}\right)=\alpha^{(1)} u_{n}\left(1-a^{(1)} u_{n}-b^{(1)} v_{n}-c^{(1)} v_{n-s_{2}}\right), \\
& f^{(2)}\left(u_{n}, v_{n}, u_{n-s_{1}}\right)=\alpha^{(2)} v_{n}\left(1-a^{(2)} u_{n}-b^{(2)} v_{n}-c^{(2)} u_{n-s_{1}}\right) .
\end{aligned}
$$

Since the above function $\left(f^{(1)}, f^{(2)}\right)$ is quasimonotone nonincreasing, the requirement on $\left(M^{(1)}, M^{(2)}\right),\left(\delta^{(1)}, \delta^{(2)}\right)$ in $(3.5)$ becomes

$$
\begin{aligned}
& \alpha^{(1)} M^{(1)}\left(1-a^{(1)} M^{(1)}-\bar{b}^{(1)} \delta^{(2)}\right) \leq 0 \leq \alpha^{(1)} \delta^{(1)}\left(1-a^{(1)} \delta^{(1)}-\bar{b}^{(1)} M^{(2)}\right), \\
& \alpha^{(2)} M^{(2)}\left(1-\bar{a}^{(2)} \delta^{(1)}-b^{(2)} M^{(2)}\right) \leq 0 \leq \alpha^{(2)} \delta^{(2)}\left(1-\bar{a}^{(2)} M^{(1)}-b^{(2)} \delta^{(2)}\right) .
\end{aligned}
$$

The above inequalities are satisfied by a sufficiently small $\left(\delta^{(1)}, \delta^{(2)}\right)>(0,0)$ and any $\left(M^{(1)}\right.$, $\left.M^{(2)}\right)$ satisfying

$$
\frac{1}{a^{(1)}}<M^{(1)}<\frac{1}{\bar{a}^{(2)}}, \quad \frac{1}{b^{(2)}}<M^{(2)}<\frac{1}{\bar{b}^{(1)}},
$$

provided that $a^{(1)}>\bar{a}^{(2)}$ and $b^{(2)}>\bar{b}^{(1)}$. 
(ii) By Theorem 4.3 , the solutions $\left(\bar{u}_{n}, \underline{v}_{n}\right),\left(\underline{u}_{n}, \bar{v}_{n}\right)$ converge to some positive equilibrium solutions $(\bar{u}, \underline{v}),(\underline{u}, \bar{v})$ that satisfy the relation

$$
\begin{array}{ll}
1-a^{(1)} \bar{u}-\bar{b}^{(1)} \underline{v}=0, & 1-\bar{a}^{(2)} \underline{u}-b^{(2)} \bar{v}=0, \\
1-a^{(1)} \underline{u}-\bar{b}^{(1)} \bar{v}=0, & 1-\bar{a}^{(2)} \bar{u}-b^{(2)} \underline{v}=0 .
\end{array}
$$

A subtraction of the above equations leads to the same relation as that in (5.8). Since $a^{(1)}>\bar{a}^{(2)}$ and $b^{(2)}>\bar{b}^{(1)}$ imply $\Delta>0$, we have $\bar{u}-\underline{u}=\bar{v}-\underline{v}=0$. This shows that $(\bar{u}, \underline{v})=$ $(\underline{u}, \bar{v}) \equiv\left(u^{*}, v^{*}\right)$, where

$$
\left(u^{*}, v^{*}\right)=\left(\frac{b^{(2)}-\bar{b}^{(1)}}{\Delta}, \frac{a^{(1)}-\bar{a}^{(2)}}{\Delta}\right),
$$

and is the unique positive equilibrium of (1.5). By Theorem 4.3, the solution $\left(u_{n}, v_{n}\right)$ corresponding to any $\left(\phi_{n}, \psi_{n}\right)$ in $\mathscr{S}$ (with $\left.\left(\phi_{0}, \psi_{0}\right)>(0,0)\right)$ converges to $\left(u^{*}, v^{*}\right)$ as $n \rightarrow \infty$.

(iii) To show the global attraction of $\left(u^{*}, v^{*}\right)$, we consider the scalar (uncoupled and without time delay) initial-value problems

$$
\begin{gathered}
U_{n}=U_{n-1}+k \alpha^{(1)} U_{n}\left(1-a^{(1)} U_{n}\right), \quad U_{0}=\phi_{0}, \\
V_{n}=V_{n-1}+k \alpha^{(2)} V_{n}\left(1-b^{(2)} V_{n}\right), \quad V_{0}=\psi_{0}(n=1,2, \ldots) .
\end{gathered}
$$

It is easily seen by a comparison between (1.5) and (5.14) that the positive solution $\left(u_{n}, v_{n}\right)$ corresponding to an arbitrary nonnegative $\left(\phi_{n}, \psi_{n}\right)$ satisfies $\left(u_{n}, v_{n}\right) \leq\left(U_{n}, V_{n}\right)$. Since by Corollary 4.2, $U_{n} \rightarrow 1 / a^{(1)}$ and $V_{n} \rightarrow 1 / b^{(2)}$ as $n \rightarrow \infty$, we see from (5.11) that there exists a large $n_{0}$ such that $u_{n} \leq M^{(1)}$ and $v_{n} \leq M^{(2)}$ for $n \geq n_{0}-\bar{s}$. On the other hand, since $\left(u_{n}, v_{n}\right)>(0,0)$ when $\left(\phi_{0}, \psi_{0}\right)>(0,0)$, there exists $n_{1} \geq n_{0}$ and a sufficiently small $\left(\delta^{(1)}, \delta^{(2)}\right)$ such that $\left(u_{n}, v_{n}\right) \geq\left(\delta^{(1)}, \delta^{(2)}\right)$ for $n_{0}-\bar{s} \leq n \leq n_{1}$. This ensures that $\left(u_{n}, v_{n}\right)$ satisfies (4.13). The convergence of $\left(u_{n}, v_{n}\right)$ to $\left(u^{*}, v^{*}\right)$ as $n \rightarrow \infty$ follows again from Theorem 4.5. This conclusion leads to the following.

Theorem 5.2. Let $a^{(1)}>\bar{a}^{(2)}, b^{(2)}>\bar{b}^{(1)}$ and $k$ satisfy condition (3.20), and let $\left(u^{*}, v^{*}\right)$ be given by (5.13). Then for any nonnegative $\left(\phi_{n}, \psi_{n}\right)$ with $\left(\phi_{0}, \psi_{0}\right)>(0,0)$, the corresponding solution $\left(u_{n}, v_{n}\right)$ of $(1.5)$ converges to $\left(u^{*}, v^{*}\right)$ as $n \rightarrow \infty$.

5.3. The prey-predator model. (i) In the prey-predator model (1.6), the reaction function $\left(f^{(1)}, f^{(2)}\right)$ is

$$
\begin{aligned}
& f^{(1)}\left(u_{n}, v_{n}, v_{n-s_{2}}\right)=\alpha^{(1)} u_{n}\left(1-a^{(1)} u_{n}-b^{(1)} v_{n}-c^{(1)} v_{n-s_{2}}\right), \\
& f^{(2)}\left(u_{n}, v_{n}, u_{n-s_{1}}\right)=\alpha^{(2)} v_{n}\left(1+a^{(2)} u_{n}-b^{(2)} v_{n}+c^{(2)} u_{n-s_{1}}\right),
\end{aligned}
$$

which is mixed quasimonotone. The requirement in (3.6) for the above function becomes

$$
\begin{aligned}
& \alpha^{(1)} M^{(1)}\left(1-a^{(1)} M^{(1)}-\bar{b}^{(1)} \delta^{(2)}\right) \leq 0 \leq \alpha^{(1)} \delta^{(1)}\left(1-a^{(1)} \delta^{(1)}-\bar{b}^{(1)} M^{(2)}\right), \\
& \alpha^{(2)} M^{(2)}\left(1+\bar{a}^{(2)} M^{(1)}-b^{(2)} M^{(2)}\right) \leq 0 \leq \alpha^{(2)} \delta^{(2)}\left(1+\bar{a}^{(2)} \delta^{(1)}-b^{(2)} \delta^{(2)}\right) .
\end{aligned}
$$


It is easy to see that the above requirement is fulfilled by a sufficiently small $\left(\delta^{(1)}, \delta^{(2)}\right)>$ $(0,0)$ and any $\left(M^{(1)}, M^{(2)}\right)$ satisfying

$$
M^{(1)}>\frac{1}{a^{(1)}}, \quad \frac{1+\bar{a}^{(2)} M^{(1)}}{b^{(2)}} \leq M^{(2)}<\frac{1}{\bar{b}^{(1)}} .
$$

The existence of $\left(M^{(1)}, M^{(2)}\right)$ satisfying the above relation is ensured if

$$
\left(\frac{\bar{b}^{(1)}}{b^{(2)}}\right)\left(\frac{1+\bar{a}^{(2)}}{a^{(1)}}\right)<1 .
$$

(ii) By Theorem 4.4, the solution $\left(\left(\bar{u}_{n}, \bar{v}_{n}\right),\left(\underline{u}_{n}, \underline{v}_{n}\right)\right)$ of (3.28), (5.15) converges to some limit $((\bar{u}, \bar{v}),(\underline{u}, \underline{v}))$ that satisfies the relation

$$
\begin{array}{ll}
1-a^{(1)} \bar{u}-\bar{b}^{(1)} \underline{v}=0, & 1+\bar{a}^{(2)} \bar{u}-b^{(2)} \bar{v}=0, \\
1-a^{(1)} \underline{u}-\bar{b}^{(1)} \bar{v}=0, & 1+\bar{a}^{(2)} \underline{u}-b^{(2)} \underline{v}=0 .
\end{array}
$$

A subtraction of the above equations leads to relation (5.8). Since condition (5.18) ensures $\Delta \neq 0$, we conclude that $\bar{u}=\underline{u}$ and $\bar{v}=\underline{v}$. This shows that $(\bar{u}, \bar{v})=(\underline{u}, \underline{v})=\left(u^{*}, v^{*}\right)$, where

$$
\left(u^{*}, v^{*}\right)=\left(\frac{b^{(2)}-\bar{b}^{(1)}}{\Delta^{*}}, \frac{a^{(1)}+\bar{a}^{(2)}}{\Delta^{*}}\right)
$$

with $\Delta^{*} \equiv a^{(1)} b^{(2)}+\bar{a}^{(2)} \bar{b}^{(1)}$. Notice from (5.18) that $\bar{b}^{(1)}<b^{(2)}$, so that $\left(u^{*}, v^{*}\right)$ is a positive equilibrium of (1.6). By Theorem 4.4, the solution $\left(u_{n}, v_{n}\right)$ corresponding to any $\left(\phi_{n}, \psi_{n}\right)$ in $\mathscr{S}$ converges to $\left(u^{*}, v^{*}\right)$ as $n \rightarrow \infty$.

(iii) Let $\left(u_{n}, v_{n}\right)$ be the solution of (1.6) corresponding to an arbitrary nonnegative $\left(\phi_{n}, \psi_{n}\right)$ with $\left(\phi_{0}, \psi_{0}\right)>(0,0)$. A comparison between the equation for $u_{n}$ in (1.6) and (5.14) for $U_{n}$ shows that $u_{n}<U_{n}$ for all $n$. This implies that there exists a large $n_{1}$ such that $u_{n} \leq M^{(1)}$ for $n \geq n_{1}$ because $U_{n} \rightarrow 1 / a^{(1)}$ as $n \rightarrow \infty$ and $1 / a^{(1)}<M^{(1)}$. Using the upper bound $M^{(1)}$ of $u_{n}$ in (1.6) for $v_{n}$, we obtain

$$
v_{n} \leq v_{n-1}+\alpha^{(2)} v_{n}\left(1+\bar{a}^{(2)} M^{(1)}-b^{(2)} v_{n}\right), \quad v_{0}=\psi_{0}
$$

This implies that there exists $n_{2}>0$ such that $v_{n} \leq\left(1+\bar{a}^{(2)} M^{(1)}\right) / b^{(2)} \leq M^{(2)}$ for $n \geq n_{2}$. The above conclusions show that $\left(u_{n}, v_{n}\right) \leq\left(M^{(1)}, M^{(2)}\right)$ for $n \geq n_{0}$ where $n_{0}=\max \left\{n_{1}, n_{2}\right\}$. It follows from the positivity of $\left(u_{n}, v_{n}\right)$ that condition $(4.13)$ holds for some $\left(\delta^{(1)}, \delta^{(2)}\right)>$ $(0,0)$. The convergence of $\left(u_{n}, v_{n}\right)$ to $\left(u^{*}, v^{*}\right)$ follows again from Theorem 4.5. In the conclusion, we have the following.

Theorem 5.3. Let conditions (5.18), (3.20) be satisfied, and let $\left(u^{*}, v^{*}\right)$ be given by (5.20). Then for any nonnegative $\left(\phi_{n}, \psi_{n}\right)$ with $\left(\phi_{0}, \psi_{0}\right)>(0,0)$ the corresponding solution $\left(u_{n}, v_{n}\right)$ of (1.6) converges to $\left(u^{*}, v^{*}\right)$ as $n \rightarrow \infty$. 
Remark 5.4. The convergence of the solution $\left(u_{n}, v_{n}\right)$ to $\left(u^{*}, v^{*}\right)$ for arbitrary nonnegative initial functions $\left(\phi_{n}, \psi_{n}\right)$ (with $\left.\left(\phi_{o}, \psi_{0}\right)>(0,0)\right)$ in Theorems 5.1, 5.2, and 5.3 shows that the positive equilibrium $\left(u^{*}, v^{*}\right)$ in the corresponding model problems (1.4), (1.5), and (1.6) is a global attractor of all positive solutions of (1.1). This implies that the trivial equilibrium solution $(0,0)$ and the semitrivial equilibrium solutions $\left(1 / a^{(1)}, 0\right),\left(0,1 / b^{(2)}\right)$ are all unstable. Notice that the condition $\left(\phi_{0}, \psi_{0}\right)>(0,0)$ is needed to ensure the convergence of $\left(u_{n}, v_{n}\right)$ to $\left(u^{*}, v^{*}\right)$ because if $\phi_{0}=0$ (or $\left.\psi_{0}=0\right)$, then the corresponding component $u_{n}$ (resp., $v_{n}$ ) is identically zero for all $n$.

\section{References}

[1] R. P. Agarwal, Difference Equations and Inequalities. Theory, Methods, and Applications, Monographs and Textbooks in Pure and Applied Mathematics, vol. 155, Marcel Dekker, New York, 1992.

[2] R. D. Driver, G. Ladas, and P. N. Vlahos, Asymptotic behavior of a linear delay difference equation, Proc. Amer. Math. Soc. 115 (1992), no. 1, 105-112.

[3] A. Drozdowicz and J. Popenda, Asymptotic behavior of the solutions of the second order difference equation, Proc. Amer. Math. Soc. 99 (1987), no. 1, 135-140.

[4] S. Elaydi, G. Ladas, J. Popenda, and J. Rakowski, Communications in difference equations, Proceedings of the 4th International Conference on Difference Equations, Poznań, 1988.

[5] M. E. Fisher, Stability of a class of delay-difference equations, Nonlinear Anal. 8 (1984), no. 6, 645-654.

[6] J. E. Franke and A. A. Yakubu, Global attractors in competitive systems, Nonlinear Anal. 16 (1991), no. 2, 111-129.

[7] K. Gopalsamy, Stability and Oscillations in Delay Differential Equations of Population Dynamics, Mathematics and Its Applications, vol. 74, Kluwer Academic Publishers, Dordrecht, 1992.

[8] I. Győri, G. Ladas, and P. N. Vlahos, Global attractivity in a delay difference equation, Nonlinear Anal. 17 (1991), no. 5, 473-479.

[9] M. P. Hassell and H. N. Comins, Discrete time models for two-species competition, Theoret. Population Biol. 9 (1976), no. 2, 202-221.

[10] V. L. Kocić and G. Ladas, Global attractivity in nonlinear delay difference equations, Proc. Amer. Math. Soc. 115 (1992), no. 4, 1083-1088.

[11] Global Behavior of Nonlinear Difference Equations of Higher Order with Applications, Mathematics and Its Applications, vol. 256, Kluwer Academic Publishers, Dordrecht, 1993.

[12] Y. Kuang, Delay Differential Equations with Applications in Population Dynamics, Mathematics in Science and Engineering, vol. 191, Academic Press, Massachusetts, 1993.

[13] R. E. Mickens, Difference Equations, Van Norstrand Reinhold, New York, 1987.

[14] C. V. Pao, Asymptotic behavior of solutions for finite-difference equations of reaction-diffusion, J. Math. Anal. Appl. 144 (1989), no. 1, 206-225.

[15]_ Nonlinear Parabolic and Elliptic Equations, Plenum Press, New York, 1992.

[16] Positive solutions and dynamics of a finite difference reaction-diffusion system, Numer. Methods Partial Differential Equations 9 (1993), no. 3, 285-311.

[17] _ Dynamics of a finite difference system of reaction diffusion equations with time delay, J. Differ. Equations Appl. 4 (1998), no. 1, 1-11.

[18] Numerical analysis of coupled systems of nonlinear parabolic equations, SIAM J. Numer. Anal. 36 (1999), no. 2, 393-416.

[19] S. Sugiyama, On the asymptotic behaviors of solutions of difference equations. I, Proc. Japan Acad. 47 (1971), 477-480. 
[20] On the asymptotic behaviors of solutions of difference equations. II, Proc. Japan Acad. 47 (1971), 481-484.

[21] Y. Takeuchi, Global Dynamical Properties of Lotka-Volterra Systems, World Scientific Publishing, New Jersey, 1996.

C. V. Pao: Department of Mathematics, North Carolina State University, Raleigh, NC 27695, USA E-mail address: cvpao@ncsu.edu 